\title{
Hybrid Unmixing Based on Adaptive Region Segmentation for Hyperspectral Imagery
}

\author{
Xiangrong Zhang, Senior Member, IEEE, Jingyan Zhang, Chen Li, Cai Cheng, Licheng Jiao, Fellow, IEEE, Huiyu
}

Zhou

\begin{abstract}
Unmixing is an important issue of hyperspectral images. Most unmixing methods adopt linear mixing models for simplicity. However, multiple scattering usually occurs between vegetation and soil in a bilinear scene. Thus, non-linear mixing problems which are difficult to be solved should be taken into consideration under this circumstance. In practice, both linear and non-linear spectral mixtures exist in hyperspectral scenes. Considering the characteristics of different regions in images, we propose a hybrid unmixing algorithm for hyperspectral images based on region adaptive segmentation (RASU). Our method uses a standard K-means clustering algorithm to obtain different regions, including homogeneous regions and detailed regions. The model of the homogeneous regions is assumed to be linear, which will be pursued using the method of sparse constrained non-negative matrix factorization (SNMF), and the mixing in the detailed regions is assumed to be based on a non-linear model. We also propose a new non-linear unmixing method, called graph regularized semi-nonnegative matrix factorization (GNMF), which considers the manifold structure of hyperspectral data as the unmixing method to deal with the detailed regions. Finally, by combining the two regions, we obtain the abundance of the whole hyperspectral image. The proposed method cannot only achieve more precise abundance, but also be good at keeping the edge information of the bilinear abundance. The experimental results on both synthetic and real data also show that the proposed method is effective for improving the unmixing accuracy of hyperspectral remote sensing images.
\end{abstract}

Index Terms-Hyperspectral unmixing, Region segmentation, Sparse constraint.

\section{INTRODUCTION}

$\mathrm{H}$ yperspectral remote sensing has been widely applied in many fields due to three unique characteristics, namely, space, radiation and spectrum. The research on hyperspectral image analysis includes feature extraction, dimensionality reduction, classification, and unmixing $[1][2][3][4]$. In the past few years, this research has obtained broad attention in the scientific community [5][6]. Due to the complexity of objects and the spatial resolution of the remote sensors used to capture the images, it is common to observe mixed pixels in a remote sensing image, especially a hyperspectral image. Therefore, hyperspectral unmixing becomes more and more important in the research community [5].

Hyperspectral unmixing refers to a process that separates the pixel spectra of a hyperspectral image into a collection of constituent spectral, or spectral signatures called endmembers and a set of fractional abundances [6]. Due to the low spatial resolution of a hyperspectral spectrometer and complexity of natural surface features, the spectrum of a single pixel cannot necessarily reflect the characteristics of a single material, and it may be a mixture of several different spectrum of materials [7]. Therefore, hyperspectral unmixing is often adopted for preprocessing hyperspectral data. Unmixing is an ill-posed inverse problem for various environmental conditions and data sets, and it is a challenging problem to solve as well [6][8].

Unmixing algorithms depend on the types of mixing [6]. Standard spectral unmixing models can be divided into two categories, linear and non-linear mixing models [9]. Linear mixing models hold when the mixing scale is macroscopic [10-13]. It should be noted that linear mixture models (LMMs) assume minimal secondary reflections and/or multiple scattering effects in the data collection procedure, and hence the measured spectra can be expressed as a linear combination of spectral signatures of the materials presented in the mixed pixels [14]. Although LMM is not always the best model to use, especially in the case of strong non-linearity, LMM is still recognized in many real-world scenarios and can be accepted due to its simplicity. Besides, the explanation of the linear analysis is straightforward.

LMMs have been widely studied and applied in recent years [15]. Traditional linear spectral unmixing algorithms usually consist of two steps: 1) endmember extraction [16], [17] and 2) abundance estimation [18-20]. For the first step, referring to as endmember extraction, a preliminary understanding of the study area should be made beforehand. Then endmembers are extracted after the numbers of endmembers are estimated and determined. The algorithms for the extraction of endmembers can be divided into the following categories: geometrical-, statistical- and sparse regression-based approaches. Vertex component analysis (VCA) [21] is a geometrical-based approach that iteratively projects data onto a direction orthogonal to the subspace spanned by the already determined endmembers. In addition, minimum volume simplex analysis (MVSA) [22] is also a geometrical-based method. With the simplex identification through variable splitting and augmented Lagrangian (SISAL) algorithms [23], it was implemented using the concept of minimum volumes. The system robustness is demonstrated by allowing the positivity constraint to be violated [6]. The sparsity-based methods have also been adopted for unmixing based on LMM in recent years. The sparse unmixing algorithm via variable splitting and augmented 
Lagrangian (SUnSAL) [24] is one of a few

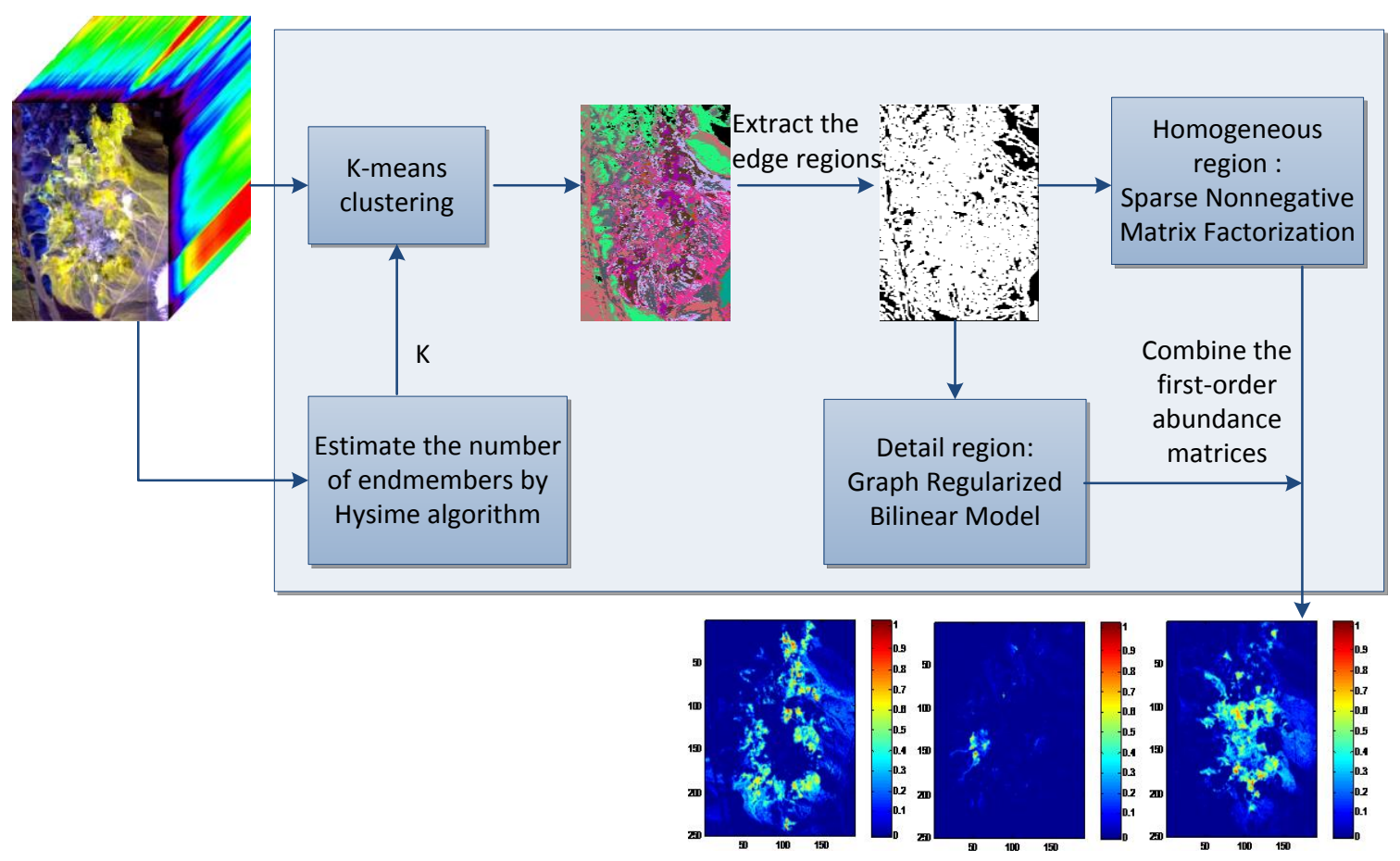

Fig. 1. The flowchart of the proposed method.

methods, generally assuming that the number of endmembers participating in each pixel is low [25]. Finally, the assumption of the collaborative SUnSAL (CLSUnSAL) [26] is that all the pixels in a hyperspectral image share the same active set of endmembers.

However, on the other hand, LMM is not a suitable approximation in some situations. Due to the complicated relationship between the actual terrain and the influence of atmospheric scattering, the spectral mixture demonstrates severe non-linearity in many real-world scenarios. Thus, we need to take a non-linear model into consideration. The generalized bilinear model (GBM) [9] is one of the most commonly used methods based on a non-linear model. It considers the second-order photon interactions between different endmembers as additional terms of a linear mixture model, assuming that the third or higher order interactions are negligible. Marinoni et al. proposed that inverting nonlinear effects starting from the measured spectral values can be achieved by assuming a harmonic description of the higher order nonlinear combinations of endmembers [27]. Moreover, Sevilla et al. [28] presented a new, computationally efficient content-based image retrieval system for hyperspectral data, which uses sparse unmixing concepts to retrieve hyperspectral scenes based on their content. Delgado et al. [29] presented parallel implementations of spatial preprocessing that have been specifically developed for commodity graphics processing units.

In addition, gradient descent algorithms (GDA) (e.g. [30]) and semi-nonnegative matrix factorization (semi-NMF) [31] are typical methods to solve the endmember problem, while the latter outperforms the former. However, the sparsity of the data has not been considered for most of the existing non-linear unmixing algorithms. Zhang et al. [32] extended the GBM incorporating the sparsity constraint of the abundance matrix with the semi-NMF by dividing the GBM into the linear and the second-order parts, which can be optimized by using an alternating optimization algorithm. Luo et al. developed a new strategy to simultaneously estimate both endmember signatures and their corresponding abundances using a bi-swarm particle swarm optimization bilinear unmixing technique based on Fan's model [33]. Recently, regularization methods have been applied to enforce the sparsity constraint on the abundance as they exploited the fact that most of the pixels are a limited number of endmembers [34]. Liu et al. [35] incorporated the characteristics of the abundance variables, namely the local spatial structural features and the statistical distribution, in the non-negative matrix factorization (NMF) to alleviate the non-convex problem of the NMF. He et al. [36] introduced a robust NMF (RNMF) model to unmix hyperspectral data by separately modeling the sparse noise and Gaussian noise that can be efficiently learned with elegant update rules. Li et al. [37] developed a new robust collaborative non-negative matrix factorization (R-CoNMF) algorithm to perform three steps of the hyperspectral unmixing chain.

Unfortunately, multiple scattering usually occurs between vegetation and soil in a bilinear scene, and hyperspectral images containing substances such as vegetation and soil may contain bilinear mixing in the border areas.

A linear spectral mixture model assumes negligible interactions among distinct ground cover materials while a nonlinear mixture model assumes that incident solar radiation is scattered within the scene itself and that these interaction events may involve several types of ground cover materials. Due to the complexity of the surface features, a single mixture 
model, either linear or nonlinear, often cannot fully reflect the complex interaction between different objects. Therefore, we use a hybrid model to simulate a real scene. First of all, we use a clustering method to cluster the actual scene, where the materials in the homogeneous region are regarded as the same substance. The material located around the edges of the homogeneous regions can be regarded as a variety of material compositions. They often have complex interactions, so it is appropriate to use a nonlinear mixture model. Finally, we combine the two parts to obtain the results.

Chen et al. [38] proposed to adopt neural networks to estimate the mixture model in hyperspectral images, and then unmix the pixels using different mixture models. However, this approach is a supervised method which required continuous human intervention. It also needs a large number of training samples, which is not an ideal solution when the training samples are unknown.

Considering the difference of several areas in a hyperspectral image, a hyperspectral image segmentation algorithm based on spectral unmixing has been developed in the literature [39]. Different from this idea, our method is a spectral unmixing method which benefits from hyperspectral image segmentation. Our method applies K-means clustering on the hyperspectral data to obtain different regions, i.e., homogeneous and detailed regions. The homogeneous regions are based on a linear mixture model, which is solved by a method of sparse constrained non-negative matrix factorization (SNMF). The detailed regions are based on a non-linear model.

Manifold learning methods have important research significance both in theory and practice. Manifold learning is to find a low dimensional manifold in a high dimensional space, and compute the corresponding embedding mapping to achieve dimension reduction. It searches for the nature of data, and finds the inherent associations that produce the data. Considering the graph constraint, we here propose graph regularized semi-nonnegative matrix factorization (GNMF) which is a new method based on semi-NMF. One of the new contributions in our approach is to considering the intrinsic manifold structure, that is, the graph constraint. Using the graph constraint, we can unmix the hyperspectral image effectively in a low-dimensional manifold.

For the detailed regions, we use the proposed non-linear unmixing method called GNMF which considers a possible manifold structure of the hyperspectral data. After combining the abundances, we finally have all the abundances of the hyperspectral image. The proposed method cannot only find more precise abundance, but also keep the edge information of the bilinear abundance. In addition, we also analyze other segmentation methods and different $\mathrm{k}$ in the $\mathrm{K}$-means clustering, which demonstrate the robustness of the proposed method. Finally, we conduct experiments on both synthetic and real data. The results indicate that the proposed method is effective and improves the unmixing accuracy of hyperspectral remote sensing images. The proposed method is depicted in Algorithm 1 and the flowchart is shown in Fig. 1.

The rest of this paper is organized as follows. In Section II, related work is presented. Section III describes the proposed algorithm based on the standard nonlinear model GBM. In Section IV, we present our novel method RASU. The experimental results and discussion on both synthetic and real data are reported in Section V. Finally, conclusions and future work are given in Section VI.

\section{II.RELATED WORK}

Generalized bilinear models, as one of the commonly used nonlinear models, have been widely used for hyperspectral image unmixing. The second-order photon interactions between different endmembers is considered as the additional terms to the linear mixture model, assuming that the third or higher order interactions are negligible [50][51]. The GBM can be written as:

$$
\mathbf{Y}=\mathbf{A X}+\mathbf{B E}+\mathbf{N}
$$

where $\mathbf{Y} \in \mathfrak{R}^{L \times P}$ is a hyperspectral data matrix with $L$ spectral bands and $P$ pixels, $\mathbf{A} \in \mathfrak{R}^{L \times K}$ is the endmember matrix of $K$ endmembers, $\mathbf{X} \in \mathfrak{R}^{K \times P}$ is the first-order abundance matrix, $\mathbf{B} \in \mathfrak{R}^{L \times K(K-1) / 2}$ is the bilinear endmember matrix, $\mathbf{E} \in \mathfrak{R}^{K(K-1) / 2 \times N_{2}}$ is the second-order abundance matrix, and $\mathbf{N} \in \mathfrak{R}^{L \times P}$ is the noise matrix. Two constraints need to be satisfied for proper unmixing: 1) the endmember and the abundance matrices are non-negative (ANC) and 2) the sum of each column of the abundance matrix is one (ASC).

However, the GBM does not take the sparsity of abundance into account, which is a significant characteristic resulting from the correlation of hyperspectral data. Since the correlation of hyperspectral data leads to the sparsity of data [24], and each pixel cannot contain all the materials, we add the sparse constraint to the abundance matrix. Considering the characteristics of sparseness and the constraints for hyperspectral unmixing, the objective function is built with the combination of reconstruction error and a sparsity measure as follows:

$$
\begin{aligned}
& \min \|\mathbf{Y}-\mathbf{A} \mathbf{X}-\mathbf{B E}\|_{F}^{2}+\lambda\|\mathbf{X}\|_{1 / 2} \\
& \text { s.t. } \quad \mathbf{X} \geq 0, \sum_{i=1}^{K}(\mathbf{X})_{i l}=1,0 \leq \mathbf{E} \leq \mathbf{X}^{*}
\end{aligned}
$$

where the first term of the objective function is the reconstruction error for the model of GBM, \|\|$_{F}$ denotes the Frobenius norm, $\lambda$ is the non-negative parameter estimated by $\lambda=\frac{1}{\sqrt{L}} \sum_{i=1}^{L} \frac{\sqrt{P}-\left\|l_{1}\right\|_{1} /\left\|l_{1}\right\|_{2}}{\sqrt{P-1}}, l_{i}$ denotes the $i$-th band in hyperspectral imagery [40]. The second term is the sparsity constraint on $\mathbf{X}$, that is, $\|\mathbf{X}\|_{1 / 2}=\sum_{k=1, p=1}^{K, P} \mathbf{x}_{p}(k)^{1 / 2}$, and $\mathbf{x}_{p}(k)$ is the abundance vector for the $k$-th endmember at the $p$-th pixel. We also have the constraints of ANC and ASC on $\mathbf{X}$. $\mathbf{X}^{*} \in \mathfrak{R}^{K(K-1) / 2 \times P}$ with each element is calculated by $\left(\mathbf{X}^{*}\right)_{(i, j) p}=(\mathrm{X})_{i p}(\mathrm{X})_{j p}(p \in\{1,2, \ldots, P\}),(\cdot)_{i j}$ denotes the value for the $i$-th row and $j$-th column of the matrix. More details of this process can be found in [29]. 


\section{GRAPH REGULARIZED BILINEAR MODEL}

Recently, existing semi-NMF algorithms have been adopted for unmixing hyperspectral images, where only Euclidean space structures are considered. In fact, hyperspectral data is more likely to be located in a low-dimensional manifold than in a high-dimensional space. Studies suggest that image data is not uniformly distributed in a high dimensional Euclidean space. Image data can be considered as the samples from the surrounding space [40] that is close to the manifold. Therefore, we consider the intrinsic manifold structure in order to properly unmix hyperspectral images.

Real hyperspectral images usually consist of several hundred spectral bands, meaning that each hyperspectral pixel can be viewed as an $L$-dimensional space. The objective of the unmixing is to find endmembers and their related proportions. To this end, matrix $\mathbf{A}$ contains a series of basic vectors in the new space. This matrix establishes a close relationship between $\mathbf{Y}$ and $\mathbf{X}$, i.e., the relation between the original image and the abundance map of different endmembers. It is natural to believe that if the spectral characteristics of two pixels are similar, this similarity can be modeled by a suitable unmixing framework. Therefore, we could have a hypothesis that if a given $L$-dimensional data point $\mathbf{Y}_{i}$ is close to $\mathbf{Y}_{j}$, the corresponding abundance $\mathbf{X}_{i}$ is also close to $\mathbf{X}_{j}$. This is a manifold assumption, which has been applied in a variety of image processing fields, such as feature learning, hyperspectral image classification [52], etc.

$\mathbf{Y}=\left[y_{1}, y_{2}, \ldots, y_{p}\right] \in \mathfrak{R}^{L \times P}$ is the matrix of hyperspectral data, each column $\left\{y_{P}\right\}_{P=1}^{P}$ of which represents a data point of the $L$-dimensional space. The weight matrix of the graph is represented as $\mathbf{W}$. If $y_{i}$ is the nearest neighbor of the $\mathrm{k}$ nearest neighbors of $y_{j}$, then the weight is specified by:

$$
\mathbf{W}_{i, j}=e^{-\frac{\left\|y_{i}-y_{j}\right\|^{2}}{\sigma^{2}}}
$$

which is known as the heat kernel [41] and $\sigma$ is the scaling parameter. When $y_{i}$ and $y_{j}$ are close, the value of $\mathbf{W}$ is relatively large. According to the above analysis, once $y_{i}$ is close to $y_{j}$, their new representations $x_{i}$ and $x_{j}$ in the new space should be close too. Thus, the following functions can be considered:

$$
\begin{aligned}
\frac{1}{2} \sum_{i, j=1}^{P}\left\|\mathbf{x}_{i}-\mathbf{x}_{j}\right\|^{2} \mathbf{W}_{i, j} & =\sum_{i=1}^{P} \mathbf{x}_{i}^{T} \mathbf{x}_{i} \mathbf{D}_{i i}-\sum_{i, j=1}^{P} \mathbf{x}_{i}^{T} \mathbf{x}_{j} \mathbf{W}_{i i} \\
& =\operatorname{Tr}\left(\mathbf{X D X} \mathbf{X}^{T}\right)-\operatorname{Tr}\left(\mathbf{X} \mathbf{W} \mathbf{X}^{T}\right) \\
& =\operatorname{Tr}\left(\mathbf{X} \mathbf{L} \mathbf{X}^{T}\right)
\end{aligned}
$$

where $\operatorname{Tr}(\cdot)$ represents the trace of the matrix, $\mathbf{X}$ is the matrix form of $\mathbf{x}_{i}$ which is the new representation of $\mathbf{y}_{i}, \mathbf{D}$ is the diagonal matrix, $\mathbf{D}_{i i}=\sum_{j} \mathbf{W}_{i j}, \mathbf{L}=\mathbf{D}-\mathbf{W}$ which is a diagonal matrix.

Then we propose GNMF by considering the graph constraint of hyperspectral unmixing. The objective function composed of errors and graph constraints is as follows:

$$
\begin{array}{ll}
\min & \frac{1}{2}\|\mathbf{Y}-\mathbf{A X}-\mathbf{B E}\|_{F}^{2}+\frac{\mu}{2} \operatorname{Tr}\left(\mathbf{X} \mathbf{L} \mathbf{X}^{T}\right) \\
\text { s.t. } & \mathbf{X} \geq 0, \sum_{i=1}^{K}(\mathbf{X})_{i l}=1,0 \leq \mathbf{E} \leq \mathbf{X}^{*}
\end{array}
$$

where the first term of the objective function is the reconstruction error based on the model of GBM, the second term is the graph constraint which considers the neighborhood consistency. Each item of $\mathbf{X}^{*} \in \mathfrak{R}^{K(K-1) / 2 \times P}$ is composed of $\left(\mathbf{X}^{*}\right)_{(i, j) p}=(\mathbf{X})_{i p}(\mathbf{X})_{j p}(p \in\{1,2, \ldots, P\}), \quad$ and $\mu$ is the non-negative parameter.

To solve Eq. (5), we adopt the method introduced in [42]. The original problem can be converted into two local optimization problems. Then, we respectively perform the iterative optimization on $\frac{1}{2}\left\|\mathbf{Y}_{11}-\mathbf{A X}\right\|_{F}^{2}+\frac{\mu}{2} \operatorname{Tr}\left(\mathbf{X} \mathbf{L} \mathbf{X}^{T}\right)$ and $\frac{1}{2}\left\|\mathbf{Y}_{22}-\mathbf{B E}\right\|_{F}^{2} \quad$ respectively, where $\quad \mathbf{Y}_{11}=\mathbf{A X}+\mathbf{N} \quad$ and $\mathbf{Y}_{22}=\mathbf{B E}+\mathbf{N} . \mathbf{X}$ is updated by:

$$
\mathbf{X} \leftarrow \mathbf{X} . *\left(\mathbf{A}^{\mathbf{T}} \mathbf{Y}_{11}+\mu \mathbf{X W}\right) . /\left(\mathbf{A}^{\mathbf{T}} \mathbf{A} \mathbf{X}+\mu \mathbf{X D}\right)
$$

and $\mathbf{E}$ is updated by:

$$
\mathbf{E}^{T} \leftarrow \mathbf{E}^{T} \cdot * \sqrt{\left(\mathbf{Y}_{22}^{T} \mathbf{B}\right)^{+}+\mathbf{E}^{T}\left(\mathbf{B}^{T} \mathbf{B}\right)^{-} /\left(\mathbf{Y}_{22}^{T} \mathbf{B}\right)^{-}+\mathbf{E}^{T}\left(\mathbf{B}^{T} \mathbf{B}\right)^{+}}
$$

ASC can be achieved by adding a row to the hyperspectral data matrix $\mathbf{Y}$ and the endmember matrix $\mathbf{A}$, which is defined as $\mathbf{Y}_{11 f}=\left[\begin{array}{c}\mathbf{Y}_{11} \\ \delta \mathbf{1}_{P}^{T}\end{array}\right]$ and $\mathbf{A}_{f}=\left[\begin{array}{c}\mathbf{A} \\ \delta \mathbf{1}_{K}^{T}\end{array}\right]$, where $\delta$ is a parameter to control the influence of ASC over the abundance matrix. Considering ASC, $\mathbf{Y}$ and $\mathbf{A}$ are replaced by $\mathbf{Y}_{11 f}$ and $\mathbf{A}_{f}$ respectively. $\mathbf{X}$ is updated by:

$$
\mathbf{X} \leftarrow \mathbf{X} . *\left(\mathbf{A}_{f}^{T} \mathbf{Y}_{11 f}+\mu \mathbf{X W}\right) . /\left(\mathbf{A}_{f}^{T} \mathbf{A}_{f} \mathbf{X}+\mu \mathbf{X D}\right)
$$

\section{HyPERSPECTRAL UNMIXING BASED ON REgION ADAPTIVE SEGMENTATION}

Due to the complexity of the actual surface features, a single mixed model: linear mixture or nonlinear mixture model, often cannot truly reflect the complex interaction between different objects. The linear model is relatively simple, and not accurate enough to meet the basic requirements of unmixing. A nonlinear mixed model is complex and difficult to describe. Therefore, we use a hybrid model to tackle the problem.

\section{A. Adaptive Region Segmentation}

Firstly, we use segmentation algorithms to divide the hyperspectral image into several parts. The aim of this step is to support the extraction of edges. We apply three segmentation algorithms, that is, standard K-means clustering, superpixels segmentation and thresholding segmentation on hyperspectral images. Because K-means clustering needs to know the number of clusters $k$, we investigate the choice of $k$ in the experimental section. Thus, we adopt the algorithm of hyperspectral signal subspace identification by minimum errors (Hysime) [43] to estimate the number of endmembers, 
which is used as $\mathrm{k}$ in the $\mathrm{K}$-means clustering. Then we label each part of the hyperspectral image after the segmentation.

According to the results of the labeling, we scan the whole image using a window of $2 \times 2$ pixels. If there is at least one different label in the window, then the pixels within the window are classified as part of the detailed regions $\mathbf{Y}_{2} \in \mathrm{R}^{L \times P_{2}}$. Otherwise, they will be classified as part of the homogeneous regions $\mathbf{Y}_{1} \in \mathrm{R}^{L \times P_{1}} . P=P_{1}+P_{2}$ denotes the total number of the samples, $P_{1}$ is the number of the samples in the homogeneous regions and $P_{2}$ is the number of the samples in the detailed regions. For example, if the labels of the pixels in the window are $1,1,1,2$ or 1, 1, 2, 3 respectively, the pixels belong to the detailed regions. If the labels of the pixels within the window are $1,1,1,1$ or $2,2,2,2$, then the pixels belong to the homogeneous regions. Then, we divide the hyperspectral image into detailed and homogeneous regions which will be processed as follows.

\section{B. Unmixing Model for a Homogeneous Region}

Considering the coexistence of linear and non-linear mixture models, we can divide a hyperspectral image into detailed and homogeneous regions. The homogeneous region is unmixed by the linear model SNMF. Then a non-negative matrix factorization algorithm, based on the Euclidean distance, is used to construct the new objective function as follows:

$$
\begin{aligned}
& \min \frac{1}{2}\left\|\mathbf{Y}_{1}-\mathbf{A} \mathbf{X}_{1}\right\|_{2}^{2}+\lambda\left\|\mathbf{X}_{1}\right\|_{1 / 2} \\
& \text { s.t. } \mathbf{X}_{1} \geq 0, \mathbf{1}^{T} \mathbf{X}_{1}=\mathbf{1}^{T}
\end{aligned}
$$

where the first term of the objective function is the reconstruction error based on the linear model, and the second term is a sparse constraint. $\mathbf{X}_{1}$ is the abundance matrix of the homogeneous regions, $\lambda$ is the parameter of the sparse regularization whose range is generally from 0.001 to 0.5 . $\mathbf{X}_{1} \geq 0, \mathbf{1}^{T} \mathbf{X}_{1}=\mathbf{1}^{T}$ are the constraints of ANC and ASC.

Using the multiplication for each iteration to optimize the objective function, we can obtain the updating formula of the homogeneous regional data. Then the endmember matrix $\mathbf{A}$ is updated by $\mathbf{A} . * \mathbf{Y}_{1} \mathbf{X}_{1}^{T} . / \mathbf{A} \mathbf{X}_{1} \mathbf{X}_{1}^{T}$, and the first-order abundance matrix $\mathbf{X}_{1}$ is updated by the formula: $\mathbf{X}_{1} * \mathbf{A}^{T} \mathbf{Y}_{1} \cdot /\left(\mathbf{A}^{T} \mathbf{A} \mathbf{X}_{1}+\frac{\lambda}{2} \mathbf{X}_{1}^{-1 / 2}\right)$, where $(\cdot)^{T}$ denotes the transpose matrix, .* and./ represent the multiplication and the division of the elements respectively. ASC is achieved by adding a row of constants to the homogeneous data matrix $\mathbf{Y}_{1}$ of the hyperspectral image and the endmember matrix $\mathbf{A}$ :
Input: a hyperspectral image

Output: abundance matrix

Step 1: estimate the number of endmembers by the Hysime algorithm as $k$

Step 2: obtain the labels using the K-means clustering

Step 3: scan the map by the window of size $2 \times 2$

if at least one different label in the window

do

Regarded as part of the detailed regions, and adopt the method of GNMF for unmixing

else

Regarded as part of the homogeneous regions, and adopt the method of SNMF for unmixing

\section{end if}

Combine the first order abundance matrices of the detailed and homogeneous regions as the first order abundance matrix. Then, select the second-order abundance matrix of the detailed regions as the nonlinear coefficient of the whole image.

$$
\mathbf{Y}_{1 f}=\left[\begin{array}{c}
\mathbf{Y}_{1} \\
\delta_{1} \mathbf{1}_{P}^{T}
\end{array}\right], \mathbf{A}_{1 f}=\left[\begin{array}{c}
\mathbf{A} \\
\delta_{1} \mathbf{1}_{K}^{T}
\end{array}\right]
$$

where $\delta_{1}$ controls the influence of the sum-to-one constraint over the abundance matrix $\mathbf{X}_{1}$. The value of $\delta_{1}$ is larger and the sum to each column of matrix $\mathbf{X}_{1}$ is closer to 1 . Let $\mathbf{Y}_{1}$ and $\mathbf{A}$ be replaced by $\mathbf{Y}_{1 f}$ and $\mathbf{A}_{1 f}$ respectively, while considering ASC, the matrix $\mathbf{X}_{1}$ is updated by the following formula:

$$
\mathbf{X}_{1} \leftarrow \mathbf{X}_{1} * \mathbf{A}_{1 f}^{T} \mathbf{Y}_{1 f} \cdot /\left(\mathbf{A}_{1 f}^{T} \mathbf{A}_{1 f} \mathbf{X}_{1}+\frac{\lambda}{2} \mathbf{X}_{1}^{-1 / 2}\right)
$$

\section{Unmixing Model for a Detailed Region}

Section III shows the detailed regions obtained by GNMF for unmixing. The new objective function can be formed as follows:

$$
\begin{array}{ll}
\min & \frac{1}{2}\left\|\mathbf{Y}_{2}-\mathbf{A} \mathbf{X}_{2}-\mathbf{B E}\right\|_{F}^{2}+\frac{\mu}{2} \operatorname{Tr}\left(\mathbf{X}_{2} \mathbf{L} \mathbf{X}_{2}^{T}\right) \\
\text { s.t. } & \mathbf{X}_{2} \geq 0, \sum_{i=1}^{K}\left(\mathbf{X}_{2}\right)_{i l}=1,0 \leq \mathbf{E} \leq \mathbf{X}_{2}^{*}
\end{array}
$$

where $\mathbf{X}_{2} \in \mathfrak{R}^{K \times P_{2}}$ is the first-order abundance matrix corresponding to the detailed regional data.

The $\mathbf{E}$ is updated by:

$$
\mathbf{E}^{T} \leftarrow \mathbf{E}^{T} \cdot * \sqrt{\left(\mathbf{Y}_{2}^{T} \mathbf{B}\right)^{+}+\mathbf{E}^{T}\left(\mathbf{B}^{T} \mathbf{B}\right)^{-} /\left(\mathbf{Y}_{2}^{T} \mathbf{B}\right)^{-}+\mathbf{E}^{T}\left(\mathbf{B}^{T} \mathbf{B}\right)^{+}}
$$

$\mathbf{X}_{2}$ is updated by:

$$
\mathbf{X}_{2} \leftarrow \mathbf{X}_{2} *\left(\mathbf{A}_{2 f}^{T} \mathbf{Y}_{2 f}+\mu \mathbf{X}_{2} \mathbf{W}\right) . /\left(\mathbf{A}_{2 f}^{T} \mathbf{A}_{2 f} \mathbf{X}_{2}+\mu \mathbf{X}_{2} \mathbf{D}\right)
$$

where $\quad \mathbf{Y}_{2 f}=\left[\begin{array}{c}\mathbf{Y}_{2} \\ \delta_{2} \mathbf{1}_{P}^{T}\end{array}\right], \quad \mathbf{A}_{2 f}=\left[\begin{array}{c}\mathbf{A} \\ \delta_{2} \mathbf{1}_{K}^{T}\end{array}\right]$ and $\delta_{2}$ controls the influence of ASC of the abundance matrix $\mathbf{X}_{2}$. It should be noted that in this paper we adopt the updated $\mathbf{A}$ of the homogeneous regions as the endmember of the detailed regions

\footnotetext{
Algorithm 1: Hyperspectral Image Unmixing Based on Region Adaptive Segmentation
} 
to keep the endmember matrix of the whole image identical. Next, the first-order abundance matrix $\mathbf{X}_{1} \in \mathfrak{R}^{K \times P_{1}}$ of the homogeneous region $\mathbf{Y}_{1}$ and the first-order abundance matrix $\mathbf{X}_{2} \in \mathfrak{R}^{K \times P_{2}}$ of the detailed region $\mathbf{Y}_{2}$ are combined as $\mathbf{X} \in \mathfrak{R}^{K \times P}$ in order to derive the first-order abundance of the whole image. The second-order abundance of the detailed region is selected as the nonlinear abundance of the whole image. Overall, the whole idea of this paper is to use the model of GNMF which considers the second-order photon interactions between different materials.

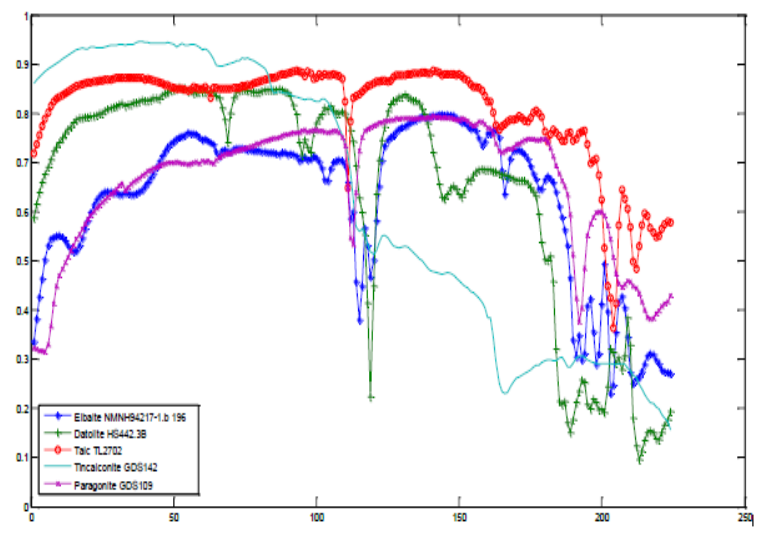

Fig. 2. Illustration of spectral features of the samples used in synthetic data.

\section{EXPERIMENT RESULTS}

In our experiments, two hyperspectral data sets are used for demonstrating the effectiveness of the proposed method. On the synthetic data, we investigate several segmentation algorithms and the choice of $\mathrm{k}$ in $\mathrm{K}$-means clustering. In addition, we perform our proposed method on two real hyperspectral data sets. The second-order abundance map of the first real data is shown and the existing unmixing algorithms are performed on the second real data for system comparison.

The quality metric adopted in our experiments to assess the unmixing results includes spectral angle distance (SAD) [45], root mean square error (RMSE) and reconstruction error (RE), which can be defined as follows:

$$
\operatorname{SAD}(\mathbf{A}, \overline{\mathbf{A}})=\arccos \left(\frac{\mathbf{A}^{\mathrm{T}} \overline{\mathbf{A}}}{\|\mathbf{A}\|\|\overline{\mathbf{A}}\|}\right)
$$

where $\mathbf{A}$ and $\overline{\mathbf{A}}$ are the matrices of the real endmember and the estimated endmember respectively. The SAD value describes the spectral angle distance between two endmember signatures, where a smaller value indicates a better estimation result [32].

$$
\mathrm{RMSE}=\left(\frac{1}{P}|\mathbf{X}-\overline{\mathbf{X}}|^{2}\right)^{\frac{1}{2}}
$$

where $\mathbf{X}$ and $\overline{\mathbf{X}}$ are the matrices of the real and estimated abundance respectively.

$$
\mathrm{RE}=\left(\frac{1}{P}|\mathbf{Y}-\overline{\mathbf{Y}}|^{2}\right)^{\frac{1}{2}}
$$

where $\mathbf{Y}$ and $\overline{\mathbf{Y}}$ are the matrices of the real and reconstructed data respectively. As for $\mathrm{SAD}$, a smaller value of RMSE and RE represents a better estimation result for the abundance map.

\section{A. Synthetic Data}

Our method is firstly validated by using the synthetic data. We use the method introduced in [44] to compose linear synthetic hyperspectral images. The linear synthetic data is made of ten spectral features presented in the U. S. Geological Survey spectral library. Fig. 2 displays five curves of spectral features which includes Elbaite NMNH94217-1b196, Datolite HS4423B, TalcTL2702, Tincalconite GDS142 and Paragonite GDS109, while the other five spectral features are not shown. We randomly select three spectral features from these ten spectral features to generate the synthetic data which is shown in Fig. 3.

In order to generate an abundance matrix that is similar to the ground truth, we use a strategy as follows. Firstly, we divide an image with the size of $z^{2} \times z^{2}$ into $z \times z$ regions. Each region is initialized with the same endmember that is randomly selected from the three endmembers. Then we apply a low-pass filter with the size of $(z+1) \times(z+1)$ on each pixel to make the pixels uniformly changed to generate the mixed data which is shown in Fig. 3(a). Secondly, we add the product of bilinear abundance and the second-order endmember matrix on Fig. 3(a) to generate the GBM-based image shown in Fig. 3(b). Fig. 3(c) is generated such that half of the pixels are generated using the generation method of Fig. 3(a) and the rest is generated using the same generation method of Fig. 3(b). It should be noticed that Fig. 3 has additive Gaussian white noise of 20 signal-to-noise ratio (SNR).

Next, we compare three segmentation algorithms on the synthetic data, i.e., the algorithms of K-means, superpixels and thresholding, which are shown in Fig. 4. The boundary maps of the different segmentation algorithms are illustrated in Fig. 5. From Figs. 4 and 5, we observe that the boundary maps of the K-means and thresholding segmentation methods generate better boundaries than the superpixels method. This is due to the over-segmentation caused by the superpixels segmentation. The thresholding segmentation is one of the most commonly used methods and can be applied to images with different gray scale ranges. There are many boundaries in the target, which may be missed, leading to unsatisfactory outcomes in a real image. Therefore, we choose K-means clustering as the segmentation method for both the synthetic and real data. For the setting of the parameters used in K-means, the clustering number $\mathrm{k}$ is set to be 3 , and the type of the distance is set to be squared Euclidean distance. We select $\mathrm{k}$ observations from data randomly and remove any clusters that have no sub-class. 

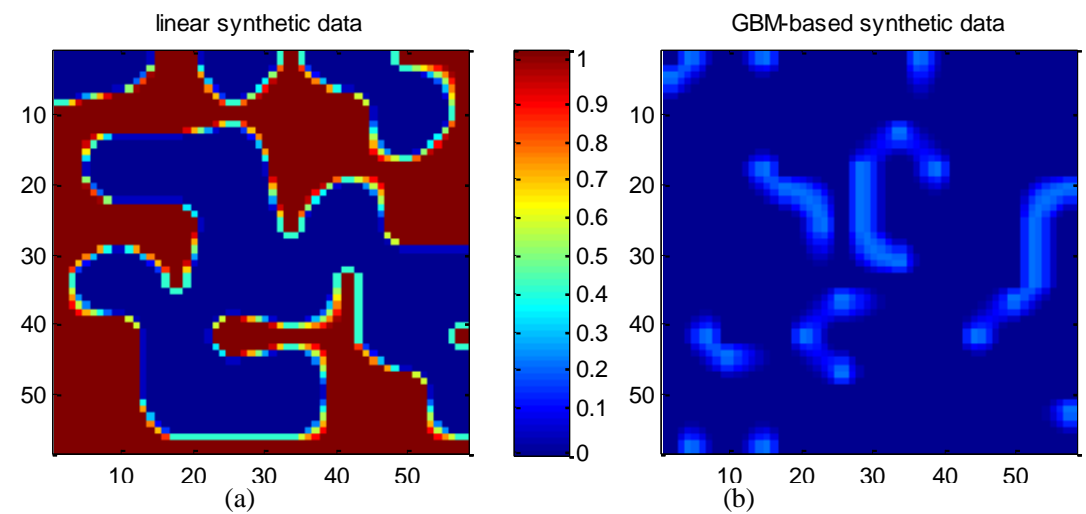

$\begin{array}{lllll}10 & 20 & 30 & 40 & 50\end{array}$

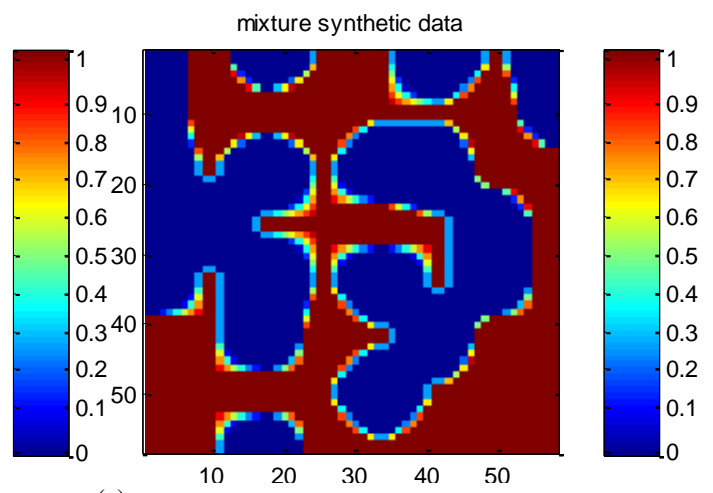

Fig. 3. The illustration of synthetic data by randomly selecting three spectral features and adding the noise with SNR=20dB. (a) linear; (b) GBM-based; (c) mixture.

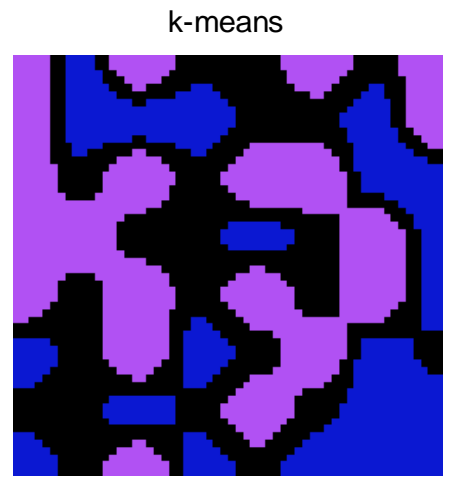

(a)

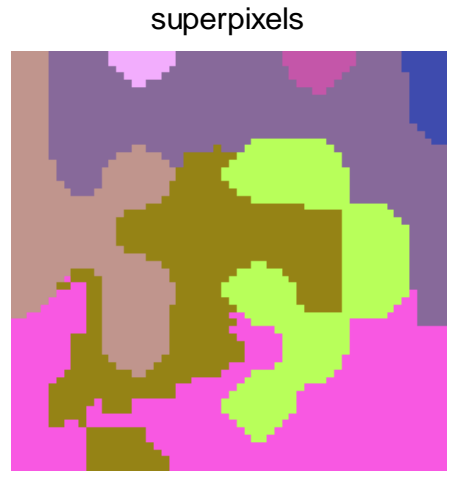

(b)

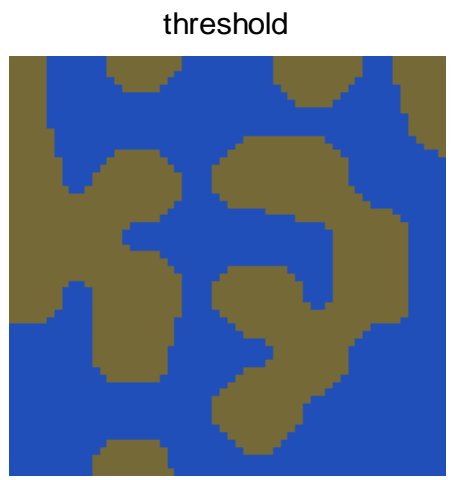

(c)

Fig. 4. The segmentation map of mixture synthetic data. (a) $k$-means $(k=2)$; (b) superpixels; (c) thresholding.

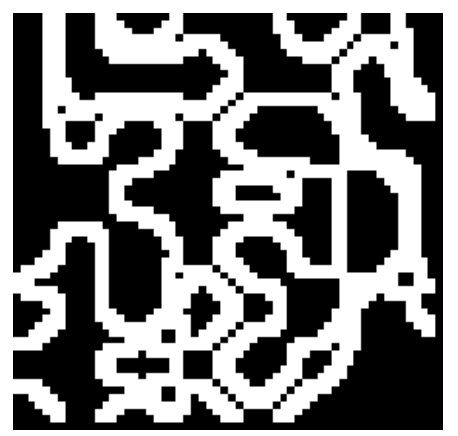

(a)

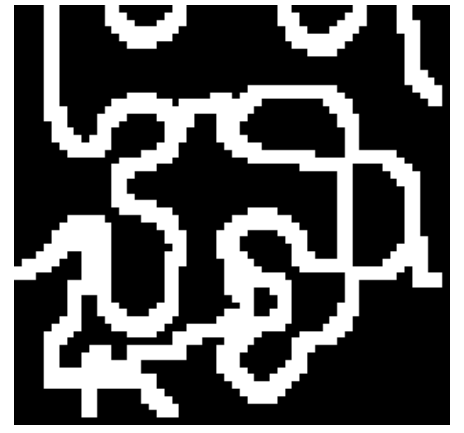

(b)

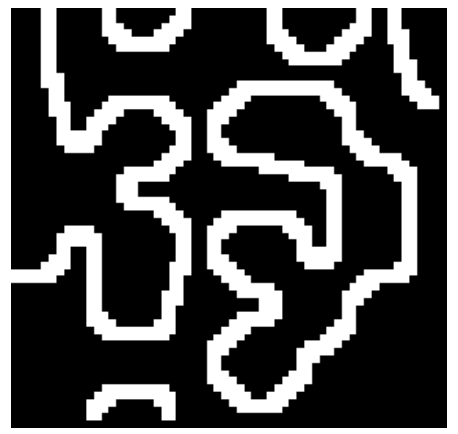

(c)

Fig. 5. The boundary map of different segmentation algorithms on mixture synthetic data. (a) $k$-means segmentation ( $k=3$ ); (b) superpixels segmentation; (c) thresholding segmentation.

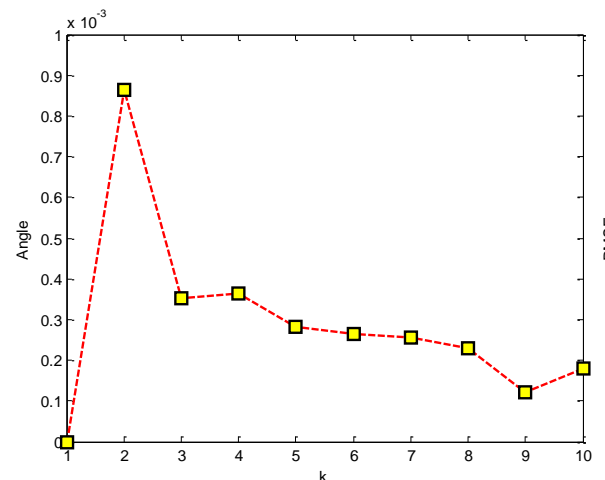

(a)

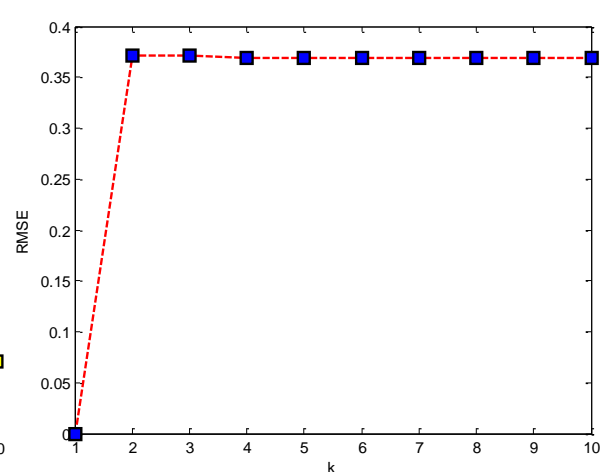

(b)

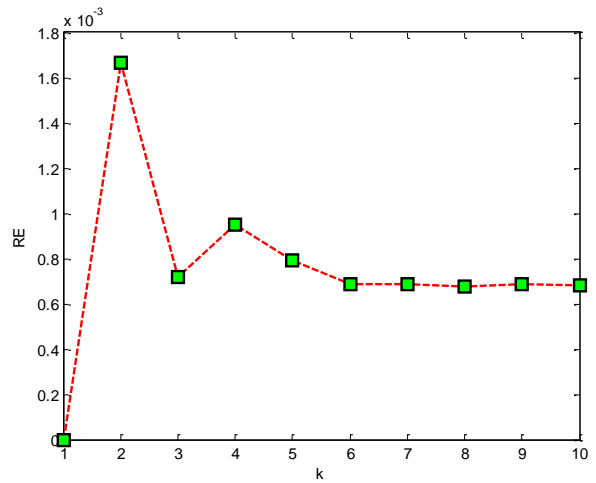

(c)

Fig. 6. The analysis to the number of $k$ in k-means clustering. (a) the value of SAD in relation to $k$; (b) the value of RMSE in relation to k; (c) the value of RE in relation to $\mathrm{k}$. 


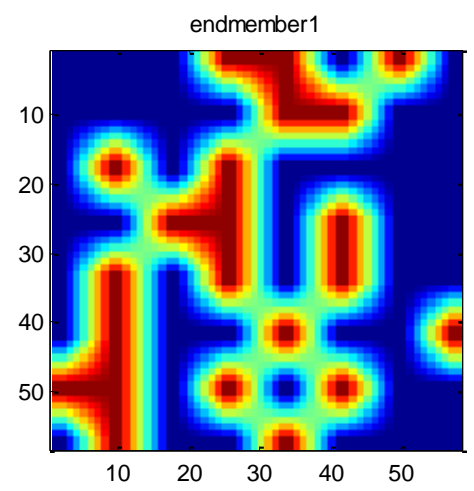

(a)
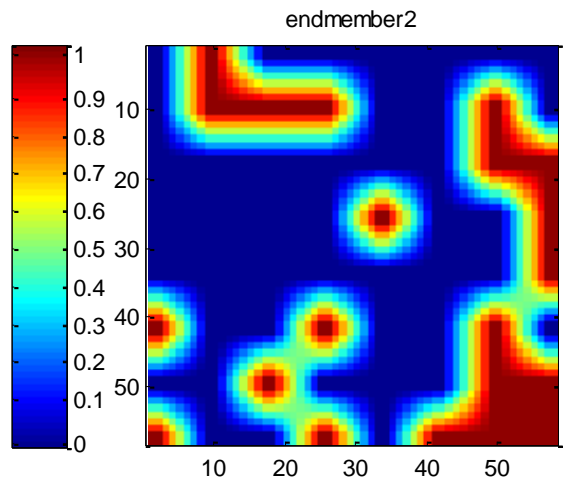

(b)
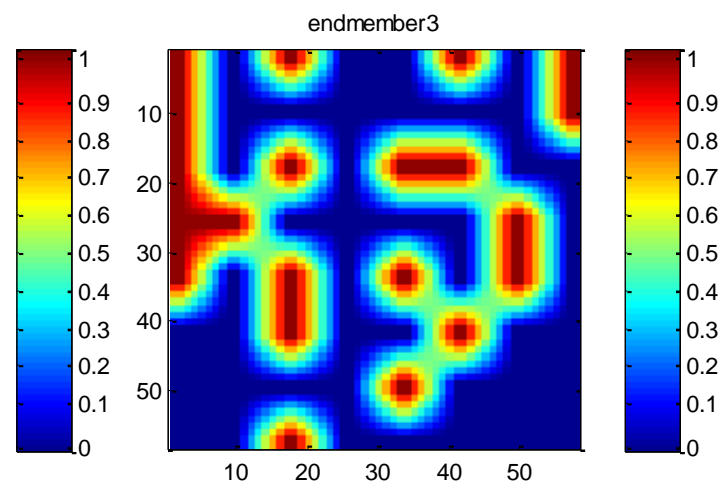

(c)

Fig. 7. The real abundance maps of three endmembers in the mixture synthetic data. (a) endmember \#1; (b) endmember \#2; (c) endmember \#3.

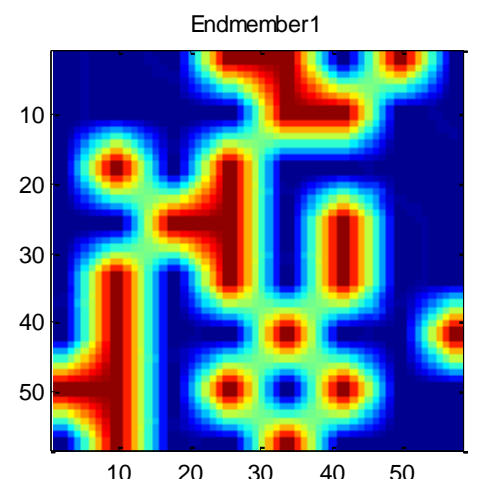

(a)

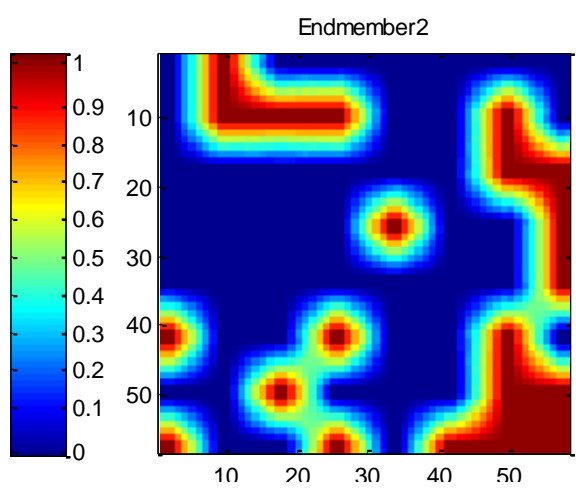

(b)

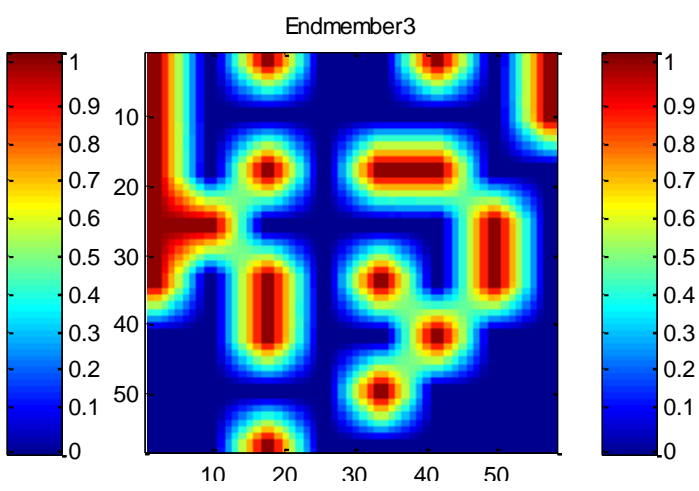

(c)

Fig. 8. The abundance maps of three endmembers generated by the proposed method on synthetic data. (a) endmember \#1; (b) endmember \#2; (c) endmember \#3.

TABLE I

RE AND RMSE VALUES AFTER APPLYING FOUR DIFFERENT UNMIXING ALGORITHMS ON SIMULATED DATA OF DIFFERENT MIXED MODELS

\begin{tabular}{c||c|c|c|c|c|c|c|c}
\hline \hline \multicolumn{1}{l||}{} & \multicolumn{4}{c|}{ RE $\left(\times 10^{-4}\right)$} & \multicolumn{4}{c}{ RMSE $\left(\times 10^{-1}\right)$} \\
\cline { 2 - 9 } & FLCS & SNMF & GNMF & RASU & FLCS & SNMF & GNMF & RASU \\
\hline \hline Fig. 3(1) & $\mathbf{1 . 6 2 8 e - 1 1}$ & $6.694 \mathrm{e}-01$ & 1.021 & 1.007 & $\mathbf{3 . 7 0 5}$ & 5.802 & 3.710 & 3.708 \\
& & & & & & & & \\
\hline Fig. 3(2) & 22.00 & 13.00 & $\mathbf{7 . 5 2 1}$ & 28 & 5.971 & 6.069 & 5.859 & $\mathbf{4 . 3 0 0}$ \\
& & & & & & & & \\
\hline Fig. 3(3) & 28.00 & 25.00 & 7.796 & $\mathbf{6 . 9 5 6}$ & 5.437 & 5.730 & 5.747 & $\mathbf{3 . 6 8 7}$ \\
\hline \hline
\end{tabular}

In addition, we also analyze the clustering number $\mathrm{k}$ of the $\mathrm{K}$-means clustering. We adopt SAD, RMSE and RE as the metrics. The curves of SAD, RMSE and RE in relations to $\mathrm{k}$ in the K-means clustering are shown in Fig. 6(a)-(c). Looking at the curves in Fig. 6, we witness that there are small fluctuations in the curve when $\mathrm{k}$ increases, and finally they are stable. The reason for this result is that, when the value of $\mathrm{k}$ increases, more and more boundaries are produced from the image. Thus, when the number of the boundaries becomes large enough, the whole image can be regarded as a boundary segmentation map. The boundaries are treated as the detailed region which is unmixed by GNMF. Finally, the errors become stable. In addition, we also find that the value between the maximum and the minimum is small. So, the proposed method is not only stable but also robust to the changes of $\mathrm{k}$. We adopt the Hysime algorithm to estimate the number of endmembers for determining the value of $\mathrm{k}$.

After applying K-means clustering on the synthetic image, 
we have two parts: homogeneous and the detailed regions. Here we perform two stages to extract endmembers and then investigate these two frames respectively.

The first stage of our work is that we use the VCA algorithm to extract the endmember matrix $\mathbf{A}$ as the initial endmember and a fully constrained least squares (FCLS) [46] solution is adopted to produce the initial abundance matrix. Then we use the SNMF to unmix in the homogeneous regions whilst extracting the updated endmember which is used as the endmember of the detailed regions. Then GNMF is used for unmixing the detailed regions. Finally, we combine the first-order abundance matrix of the detailed regions and the homogeneous areas as the first-order abundance matrix of the whole image. Then we select the second-order abundance matrix of the detailed regions as the nonlinear abundance of the whole image. The whole idea of this paper is to use the model of GBM which considers the second-order photon interactions between different materials. Figs. 7 and 8 show the truth abundance maps and the estimated abundance maps of the three endmembers. The more similarity between Figs. 7 and Fig. 8 is, the performance of the proposed method is better. Figs. 7 and 8 look similar, which show the effectiveness of our method. From these two figures, we recognize that the proposed method can achieve a better unmixing result. In addition, Table I shows the values of RE and RMSE on the synthetic data. There are three kinds of synthetic data: linear, nonlinear and mixed data. In Table I, the best results of three different kinds images are in bold which makes Table I more readable. From Table I, we can find that the proposed method can achieve the minimum value of RE for Fig. 3(c). It shows the effectiveness of our proposed method for the mixture data. The reason is that we consider the intrinsic manifold structure to unmix the hyperspectral image which is more likely located in a low-dimensional manifold. The method of GNMF achieves the minimum value for Fig.3(b) compared with the other three methods, which shows the effectiveness of the GNMF for the nonlinear data. On the other hand, from the values of RMSE on the synthetic data, we can see that the FCLS is the best method for Fig. 3(a) which is slightly better than our method. The main reason is that Fig. 3(a) is the outcome of undertaking linear synthesis of spectra. The algorithm of FCLS is based on a linear mixture model. Thus, FCLS can achieve better results for this type of data. In addition, the proposed method can obtain the best result in both Fig. 3(b) and (c). Overall, the proposed method can obtain a beneficial effect for unmixing because we use a hybrid model to simulate the real scenes, which takes the complexity of the actual surface features into account.

The second stage of this work is that we use the VCA algorithm to extract endmembers of the homogeneous and detailed regions respectively. Then we adopt SNMF and GNMF to unmix separately and do not combine the abundance matrices at the end of the algorithm. Finally, we analyze the results of the homogeneous and detailed region separately. For the experiments shown in Fig. 3(c), we obtain that the values of $\mathrm{RE}$ and RMSE in the homogeneous regions are $2.2190 \mathrm{e}-4$ and
0.4296 respectively, which are lower than the results of FCLS, $6.7939 \mathrm{e}-4$ and 0.6345 . The values of RE and RMSE in the detailed regions are 0.0114 and 0.3359 which are lower than those of FCLS, i.e., 0.1964 and 0.4613 . This also shows that our method is more effective than the others for unmixing.

Overall, the main difference between the two stages is the endmembers between the homogeneous and the detailed regions. At the first stage, we have the same endmembers on these two different regions which are updated by SNMF to combine the abundance matrices at the end of the algorithm. At the 2nd stage, we use different endmembers from the homogeneous and the detailed regions and their corresponding abundance matrices will not be combined in the end.

\section{B. Real Data}

The first real hyperspectral data in our experiments was taken in 1997, i.e., Moffet Field, which is at the southern end of San Francisco Bay. The image contains all 224 bands that the AVIRIS sensor collected with a wavelength spectrum from 400 to 2500 nanometers that covers the complete VIS-NIR-SWIR spectrum. This dataset has been widely studied in the remote sensing community [47]. A region of $160 \times 250$ pixels of the original image is used in our experiments, which is shown in Fig. 9(a). After removing the channels affected by dense water vapor and atmospheric, we use the remaining 189 channels, which is a common preprocess for the analysis of the hyperspectral data. Specifically, there are three endmembers in the image, i.e., soil, vegetation and water respectively [48]. The maps of K-means clustering and the boundary are respectively shown in Fig. 9(b) and (c). We adopt the Hysime algorithm to estimate the number of endmembers which uses 3 as the value of k. Fig. 10 shows the first-order abundance map of three different endmembers in Moffet Field by our proposed method and we also show the second-order abundance maps separately in Fig. 11(a)-(c).

In our real data experiments, the second image is the well-known AVIRIS Cuprite data set in west-central Nevada in 1997 which is shown in Fig. 12(a). The portion used in our experiments corresponds to a $250 \times 191$ subset of the data. Due to water absorption and low SNR, the number of the spectral bands has been reduced from 224 to 188 . As for the other two data sets, we firstly get the number of $\mathrm{k}$ by the Hysime method which is equal to 14. Fig. 12(b) and (c) respectively show the maps of the K-means clustering and the boundary of the Cuprite data. We also compare the proposed method with the other unmixing methods including the algorithms such as SUnSAL and CLSUnSAL. As shown in Fig. 13, due to the limited space in this paper, there are only three endmembers and their spectral features curves are shown including the alunite, the buddingtonite and the chalcedony which are known to be present (in prominent fashion) in the Cuprite mining district [49]. Most of the experimental results presented in this paper show the effectiveness of the proposed method for hyperspectral image unmixing. 


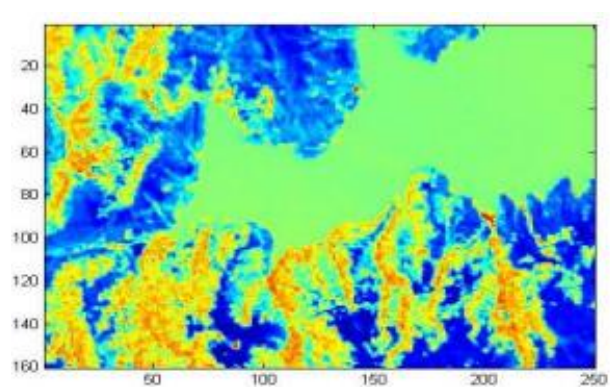

(a)

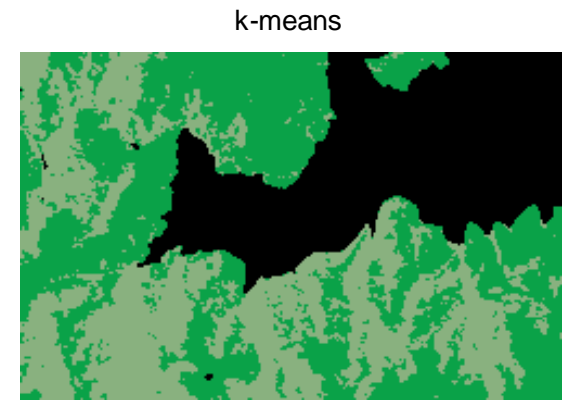

(b)

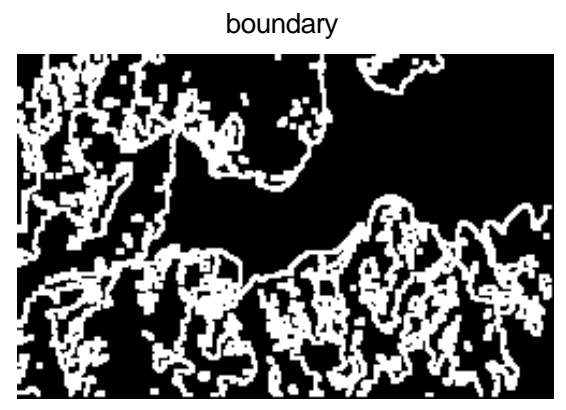

(c)

Fig. 9. The Moffet field and its maps of segmentation by k-means clustering and boundary detection. (a) the map of Moffeit field; (b) the segmentation map of k-means clustering $(\mathrm{k}=3)$; (c) the boundary map.

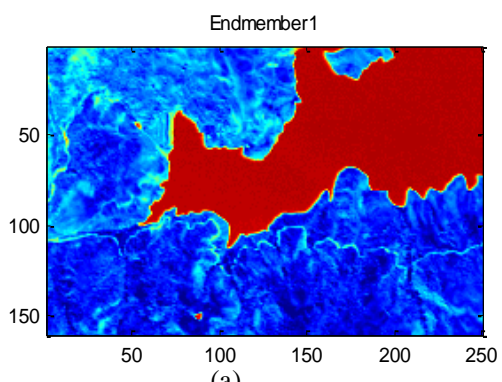

(a)

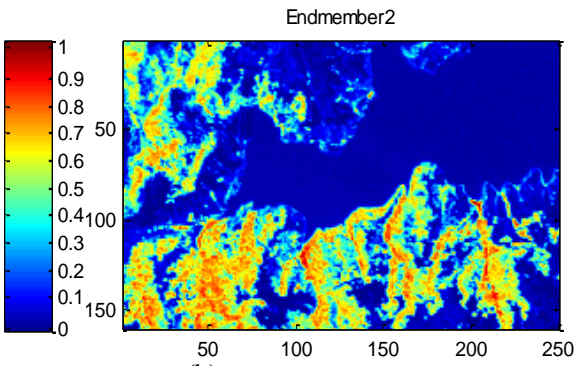

(b)

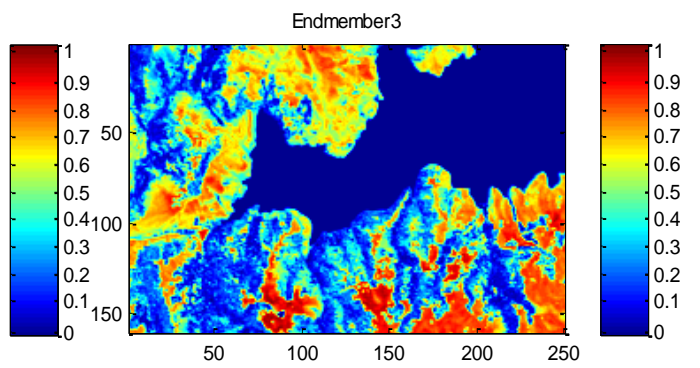

(c)

Fig. 10. The first-order abundance maps of the Moffet field generated by the proposed method. (a) the abundance map of water; (b) the abundance map of vegetables; (3) the abundance map of soil.

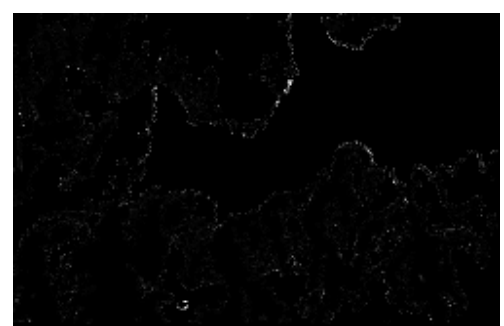

(a)

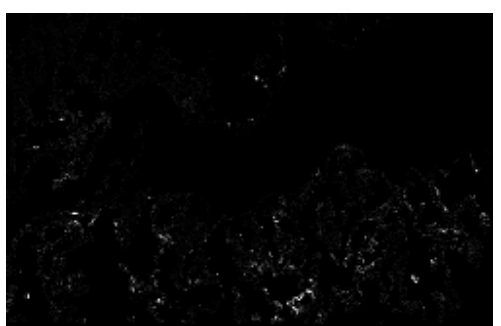

(b)

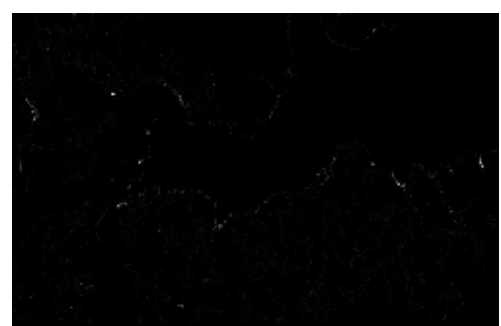

(c)

Fig. 11. The second-order abundance maps of the Moffet field generated by the proposed method. (a) the second-order abundance map of water-vegetables; (b) the second-order abundance map of vegetables-soil; (c) the second-order abundance map of soil-water.

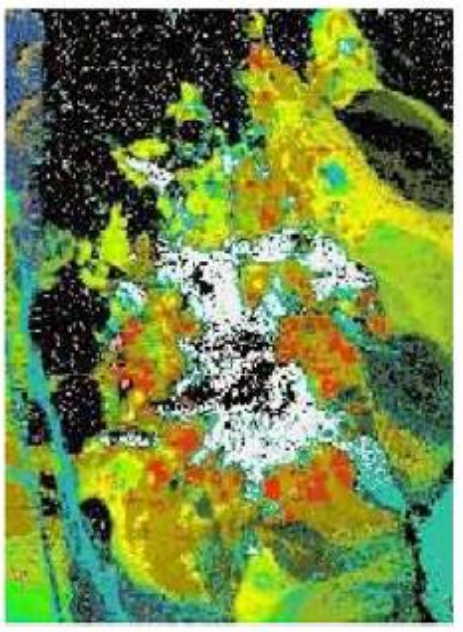

(a)

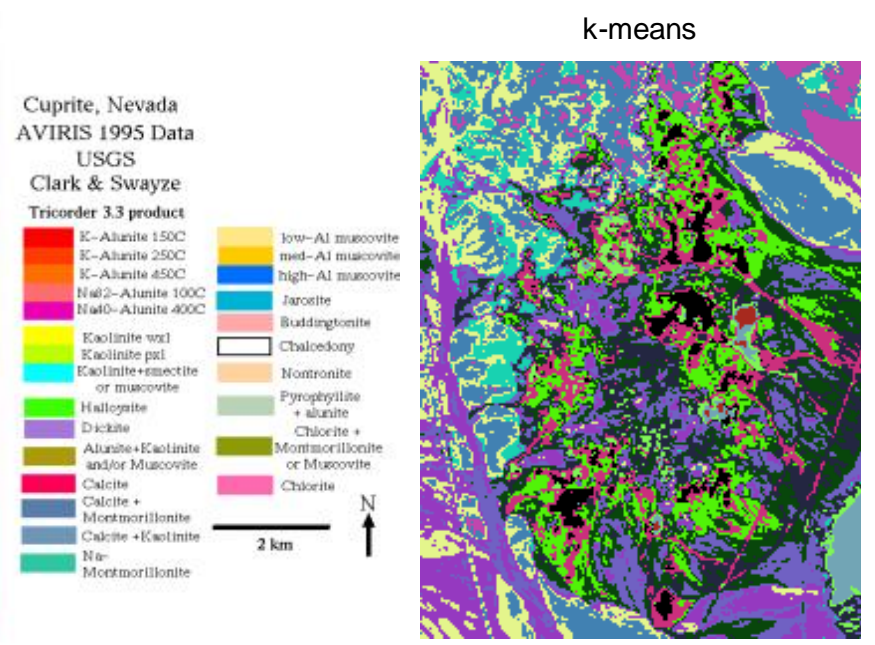

(b)

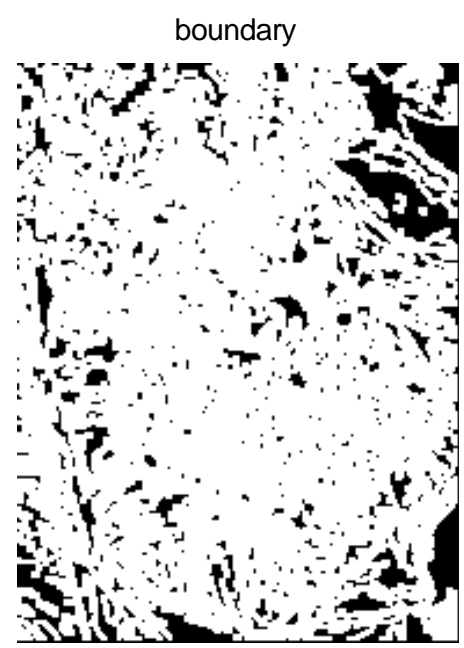

(c)

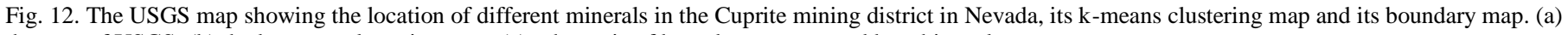
the map of USGS; (b) the k-means clustering map; (c) schematic of boundary represented by white color. 

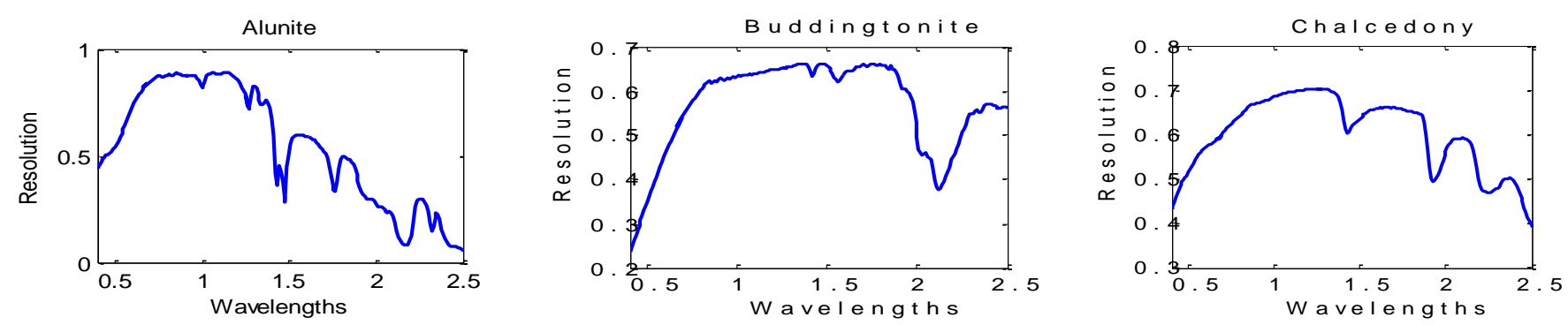

SUnSAL

CLSUnSAL

RASU
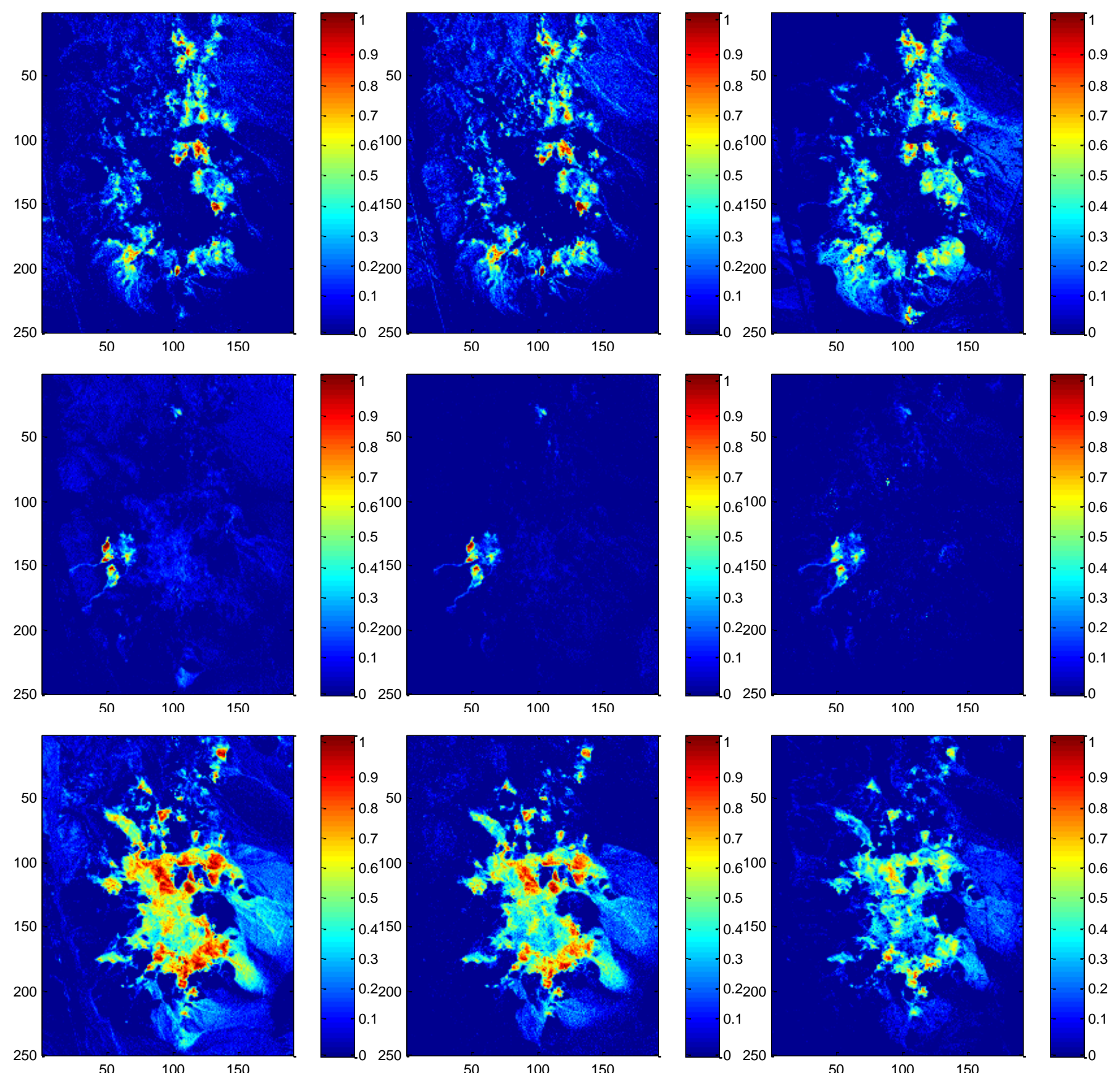

Fig. 13. Abundance maps estimated for the minerals: alunite, buddingtonite and chalcedony by SUnSAL, CLSUnSAL and RASU algorithms of Cuprite data. 


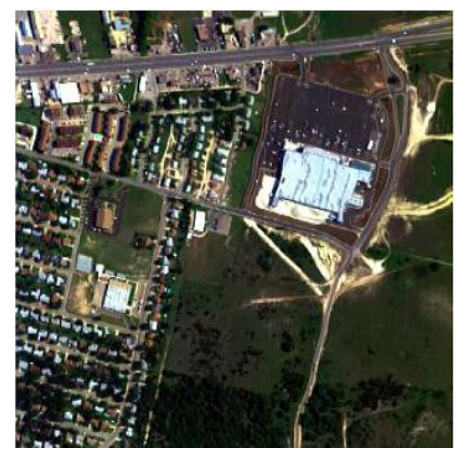

(a)

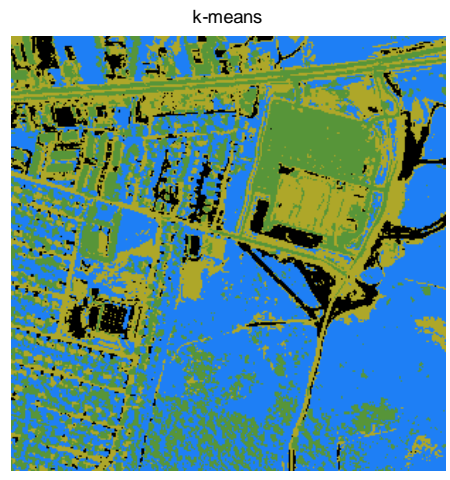

(b)

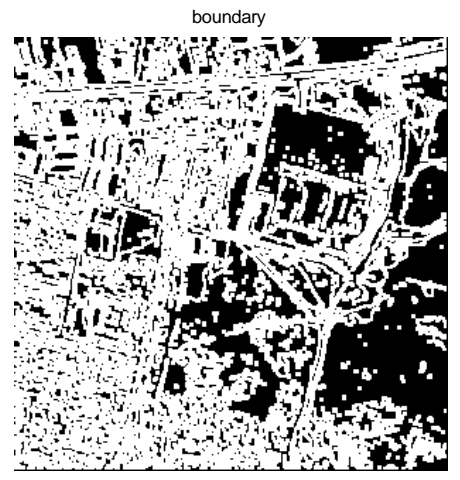

(c)

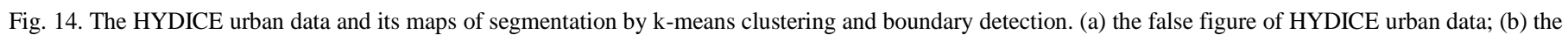
segmentation map of k-means clustering $(\mathrm{k}=4)$; (c) the boundary map represented by white color.

$\mathrm{L}_{1 / 2}-\mathrm{RNMF}$
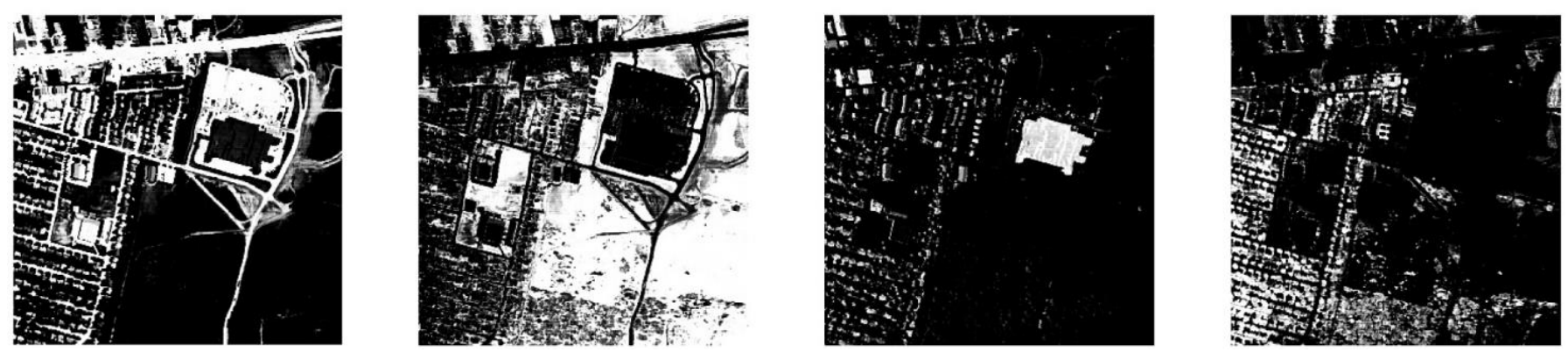

SUnSAL
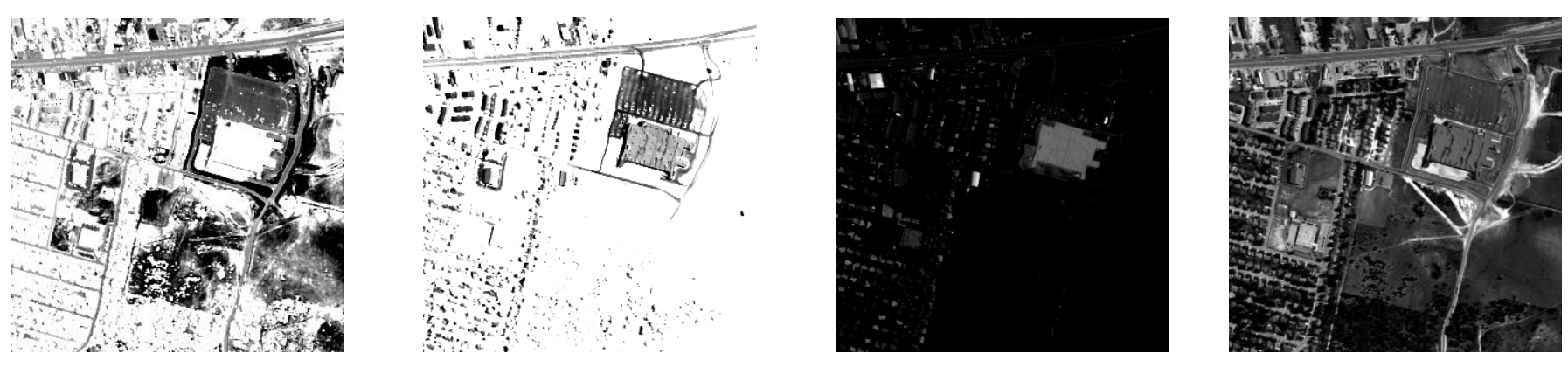

RASU

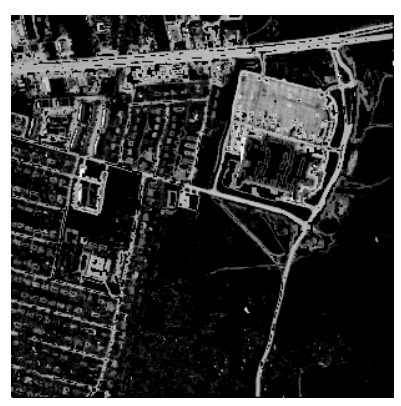

(a)

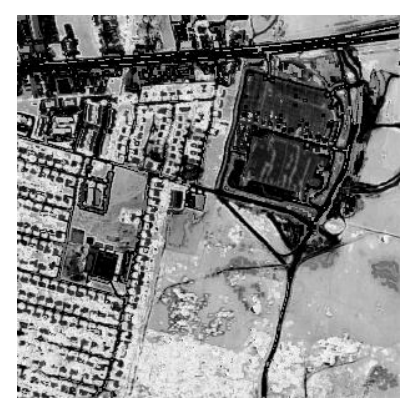

(b)

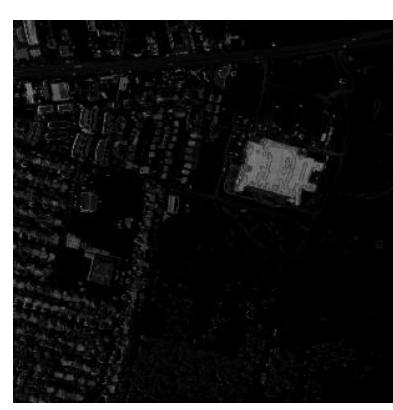

(c)

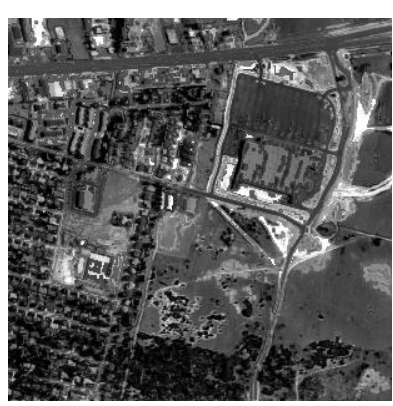

(d)

Fig. 15. Abundance maps estimated for the minerals: roof, grass, asphalt and tree by RASU algorithms of the HYDICE urban data. (a) Asphalt; (b) Grass; (c) roof; (d) Tree. 
TABLE II

SAD VALUES OF THE DIFFERENT ALGORITHMS FOR HYDICE URBAN DATA

\begin{tabular}{|c|c|c|c|c|}
\hline & RASU & VCA & L $_{1 / 2}-\mathrm{NMF}$ & $\mathrm{L}_{1 / 2}-\mathrm{RNMF}$ \\
\hline Asphalt & 1.0717 & 1.1444 & 0.0865 & 0.0871 \\
\hline Grass & 0.3280 & 0.6598 & 0.0864 & 0.0838 \\
\hline Roof & 1.4503 & 0.3601 & 0.1596 & 0.0590 \\
\hline Tree & 0.9603 & 0.7588 & 0.0934 & 0.0811 \\
\hline Mean & 0.9526 & 0.7308 & 0.1075 & 0.0777 \\
\hline
\end{tabular}

TABLE III

FLOATING-POINT CALCULATION AT EACH ITERATION BY NMF, SNMF AND GNMF.

\begin{tabular}{|c|c|c|c|c|c|c|c|}
\hline \multirow{2}{*}{ Region } & \multirow{2}{*}{ Methods } & \multicolumn{3}{|c|}{ Update A } & \multicolumn{3}{|c|}{ Update $\mathbf{X}$} \\
\hline & & Addition & Multiplication & Division & Addition & Multiplication & Division \\
\hline \multirow{2}{*}{ Homogeneous } & NMF & $\begin{array}{c}\mathrm{LP}_{1} \mathrm{~K}+\left(\mathrm{L}+\mathrm{P}_{1}\right) \\
\mathrm{K}^{2}\end{array}$ & $\begin{array}{c}\mathrm{LP}_{1} \mathrm{~K}+\left(\mathrm{L}+\mathrm{P}_{1}\right) \mathrm{K}^{2}+ \\
\mathrm{LK}\end{array}$ & LK & $\mathrm{LP}_{1} \mathrm{~K}+\left(\mathrm{L}+\mathrm{P}_{1}\right) \mathrm{K}^{2}$ & $\mathrm{LP}_{1} \mathrm{~K}+\left(\mathrm{L}+\mathrm{P}_{1}\right) \mathrm{K}^{2}+\mathrm{P}_{1} \mathrm{~K}$ & $\mathrm{P}_{1} \mathrm{~K}$ \\
\hline & SNMF & $\begin{array}{c}\mathrm{LP}_{1} \mathrm{~K}+\left(\mathrm{L}+\mathrm{P}_{1}\right) \\
\mathrm{K}^{2}\end{array}$ & $\begin{array}{c}\mathrm{LP}_{1} \mathrm{~K}+\left(\mathrm{L}+\mathrm{P}_{1}\right) \mathrm{K}^{2}+ \\
\mathrm{LK}\end{array}$ & LK & $\begin{array}{c}(\mathrm{L}+1) \mathrm{P}_{1} \mathrm{~K}+\left(\mathrm{L}+\mathrm{P}_{1}+1\right) \mathrm{K}^{2}+ \\
\mathrm{P}_{1} \mathrm{~K}\end{array}$ & $\begin{array}{c}(\mathrm{L}+1) \mathrm{P}_{1} \mathrm{~K}+\left(\mathrm{L}+\mathrm{P}_{1}+1\right) \mathrm{K}^{2}+ \\
\mathrm{P}_{1} \mathrm{~K}\end{array}$ & $\mathrm{P}_{1} \mathrm{~K}$ \\
\hline \multirow{2}{*}{ Detailed } & NMF & $\begin{array}{c}\mathrm{LP}_{2} \mathrm{~K}+\left(\mathrm{L}+\mathrm{P}_{2}\right) \\
\mathrm{K}^{2}\end{array}$ & $\begin{array}{c}\mathrm{LP}_{2} \mathrm{~K}+\left(\mathrm{L}+\mathrm{P}_{2}\right) \mathrm{K}^{2}+ \\
\mathrm{LK}\end{array}$ & LK & $\left(\mathrm{L}+\mathrm{P}_{2}\right) \mathrm{K}^{2}+\mathrm{LP}_{2} \mathrm{~K}$ & $\left(\mathrm{~L}+\mathrm{P}_{2}\right) \mathrm{K}^{2}+(\mathrm{L}+1) \mathrm{P}_{2} \mathrm{~K}$ & $\mathrm{P}_{2} \mathrm{~K}$ \\
\hline & GNMF & ----- & ------ & ------ & $\begin{array}{c}\left(\mathrm{L}+\mathrm{P}_{2}+1\right) \mathrm{K}^{2}+(\mathrm{L}+1) \mathrm{P}_{2} \mathrm{~K}+ \\
2 \mathrm{P}_{2}{ }^{2} \mathrm{~K}\end{array}$ & $\begin{array}{c}\left(\mathrm{L}+\mathrm{P}_{2}+1\right) \mathrm{K}^{2}+(\mathrm{L}+3) \mathrm{P}_{2} \mathrm{~K}+ \\
2 \mathrm{P}_{2}^{2} \mathrm{~K}\end{array}$ & $\mathrm{P}_{2} \mathrm{~K}$ \\
\hline
\end{tabular}

In the real data experiments, the third image is the HYDICE urban data set which was collected by the HYDICE sensor with a wavelength spectrum from 400 to 2500 nanometers. Its false figure is shown in Fig. 14(a). There are 210 bands in the Urban dataset with the size of $307 \times 307$ pixels. After removing the channels affected by dense water vapor and atmospheric, we use the remaining 162 channels. There are four endmembers in the image, i.e., asphalt, grass, roof and tree respectively. Fig. 14(b) and (c) show the maps of the segmentation by k-means clustering and boundary detection.

We also compare the proposed method with another unmixing method called $\mathrm{L}_{1 / 2}$-RNMF [36]. The abundance maps of the proposed method and the $\mathrm{L}_{1 / 2}$-RNMF for HYDICE urban dataset, shown in Fig. 15. From Fig.15, we can see that the results of our method show more details than the method of $\mathrm{L}_{1 / 2}$ - RNMF. The reason is that we consider the intrinsic manifold structure, that is, the graph constraint, and the complexity of the actual surface features that hyperspectral images not only contain the linear spectral mixtures, but also the non-linear spectral mixture. In addition, we also study the values of SAD from the different algorithms whose results are shown in Table II. The $\mathrm{SAD}$ values of $\mathrm{L}_{1 / 2} \mathrm{NMF}$ and $\mathrm{L}_{1 / 2}-\mathrm{RNMF}$ come from [36]. From Table II, we can find that the results of the proposed method are comparable to the other three methods of endmember extraction. We extract the endmembers of the whole hyperspectral image firstly. Then we refresh the endmembers in the detailed regions and use these refreshed endmembers as the endmembers used in the homogeneous regions in order to combine the results of the detailed and homogeneous regions in the end. This operation results in the consequence that the endmembers are not accurate, and they may better suit the homogeneous regions.
Besides, we also present the floating-point calculation in SNMF and GNMF compared with the traditional NMF, which is shown in Table III. Due to the existing of different regions, we can compare the proposed method with NMF respectively in homogeneous and detailed regions. From Table III, we can find that the calculation of GNMF is less for that we used the endmembers extracted before in the homogeneous regions which will make the SAD high.

\section{CONCLUSION}

Due to the complexity of the actual surface features, hyperspectral images not only contain the linear spectral mixtures, but also the non-linear spectral mixture. However, most of the unmixing methods for hyperspectral images only consider the existence of a linear mixture model or a non-linear mixture model. In this paper, we consider the coexistence of the linear spectral mixture model and the non-linear spectral mixture model, and we have introduced a hyperspectral image unmixing method based on adaptive region segmentation. According to the characteristics of different regions generated by the segmentation of K-means clustering, we obtain accurate abundances and keep the edge information of the bilinear abundance by using the SNMF and the GNMF in two different regions. After combining the abundance, we obtain the whole abundance in the hyperspectral image. In addition, we also proposed a new method based on a nonlinear model called GNMF. The experiments show that the proposed method is promising for hyperspectral image unmixing. 


\section{ACKNOWLEDGMENT}

This work was supported by the National Natural Science Foundation of China (Nos. 61772400, 61501353, 61772399, 91438201, 61573267). Dr H. Zhou is supported by UK EPSRC under Grants EP/N508664/1, EP/R007187/1 and EP/N011074/1, and Royal Society-Newton Advanced Fellowship under Grant NA160342.

\section{REFERENCES}

[1] A. J. Brown, "Spectral Curve Fitting for Automatic Hyperspectral Data Analysis." IEEE Transactions on Geoscience and Remote Sensing, vol. 44, no. 6, pp: 1601-1608, 2006.

[2] S. Li, T. Lu, L. Fang, X. Jia, J. A. Benediktsson: Probabilistic Fusion of Pixel-Level and Superpixel-Level Hyperspectral Image Classification. IEEE Trans. Geoscience and Remote Sensing54(12): 7416-7430 (2016)

[3] X. Jia, B.C. Kuo, and M. M. Crawford, "Feature mining for hyperspectral image classification," Proceedings of the IEEE, vol. 101, pp. 676 - 697, 2013.

[4] X. Zhang, Q. Song, R. Liu, W. Wang, and L. Jiao, "Modified co-training with spectral and spatial views for semisupervised hyperspectral image classification," IEEE Journal of Selected Topics in Applied Earth Observations and Remote Sensing, vol. 7, no. 6, pp. 2044-2055, 2014.

[5] A. Plaza, Q. Du, J. Bioucas-Dias, X. Jia, and F. Kruse, "Foreword to the special issue on spectral unmixing of remotely sensed data," IEEE Trans.Geosci. Remote Sens., vol. 49, no. 11, pp. 4103-4110, Nov. 2011.

[6] J. M. Bioucas-Dias, A. Plaza, N. Dobigeon, M. Parente, Q. Du, P. Gader, and J. Chanussot, "Hyperspectral unmixing overview: Geometrical, statistical, and sparse regression -based approaches," IEEE J. Sel. Topics Appl. Earth Observ., vol. 5, no. 2, pp. 354-379, Apr. 2012.

[7] N. Keshava, "A survey of spectral unmixing algorithms," Lincoln Lab. J., vol. 14, no. 1, pp. 55-78, Jan. 2003.

[8] N. Keshava and J. F. Mustard, "Spectral unmixing," IEEE Signal Process. Mag., vol. 19, no. 1, pp. 44-57, Jan. 2002.

[9] N. Dobigeon, Jean-Yves Tourneret, C. Richard, J. Carlos, M. Bermudez, S. McLaughlin, and A. O. Hero, "Nonlinear unmixing of hyperspectral images," IEEE Signal Processing Magazine, pp.82-94, Jan. 2014.

[10] J. B. Adams, M. O. Smith, and P. E. Johnson, "Spectral mixture modeling: A new analysis of rock and soil types at the Viking Lander 1 site," J.Geophys. Res., vol. 91, no. B8, pp. 8098-8112, Jul. 1986.

[11] J. J. Settle and N. A. Drake, "Linear mixing and the estimation of ground cover proportions," Int. J. Remote Sens., vol. 14, no. 6, pp. 1159-1177, Apr. 1993.

[12] M. Petrou and P. G. Foschi, "Confidence in linear spectral unmixing of single pixels," IEEE Trans. Geosci. Remote Sens., vol. 37, no. 1, pp. 624-626, Jan. 1999.

[13] J. Settle, "On the effect of variable endmember spectra in the linear mixture model," IEEE Trans. Geosci. Remote Sens., vol. 44, no. 2, pp. 389-396, Feb. 2006.

[14] Antonio Plaza and Javier Plaza, "Parallel Implementation of Linear and Nonlinear Spectral Unmixing of Remotely Sensed Hyperspectral Images," SPIE Remote Sensing, 8183(1): 187-193, 2011.

[15] Jun Li et al., "Minimum Volume Simplex Analysis: A Fast Algorithm for Linear Hyperspectral Unmixing," IEEE Trans. Geosci. Remote Sens., vol. 53, no. 9, pp. 5067-5082, Sep. 2015.

[16] A. Plaza, P. Martinez, R. Perez, and J. Plaza, "A quantitative and comparative analysis of endmember extraction algorithms from hyperspectral data," IEEE Trans. Geosci. Remote Sens., vol. 42, no. 3, pp. 650-663, Mar. 2004.

[17] Q. Du, N. Raksuntorn, N. Younan, and R. King, "Endmember extraction for hyperspectral image analysis," Appl. Opt., vol. 47, no. 28, pp. 77-84, Jul. 2008.

[18] D. Heinz and C.I. Chang, "Fully constrained least squares linear mixture analysis for material quantification in hyperspectral imagery," IEEE Trans. Geosci. Remote Sens., vol. 39, no. 3, pp. 529-545, Mar. 2001.

[19] A. J. Brown, S. J. Hook, A. M. Baldridge, J. K. Crowley, N. T. Bridges, B. Bridges, B. J. Thomson, G. M. Marion, C. R. S. Filho, J. L. Bishop, "Hydrothermal Formation of Clay-Carbonate Alteration Assemblages in the Nili Fossae Region of Mars." Earth and Planetary Science Letters 297, 297, pp: $174-82,2010$
[20] A. J. Brown, B. Sutter, and S. Dunagan. "The MARTE Imaging Spectrometer Experiment: Design and Analysis.” Astrobiology 8, no. 5, pp: $1001-1011,2008$.

[21] J. M. P. Nascimento and J. M. Bioucas-Dias, "Vertex component analysis: A fast algorithm to unmix hyperspectral data," IEEE Trans. Geosci. Remote Sens., vol. 43, no. 4, pp. 898-910, 2005.

[22] J. Li and J. Bioucas-Dias, "Minimum volume simplex analysis: A fast algorithm to unmix hyperspectral data," IEEE Int. Conf. Geosci. Remote Sens, vol. 3, pp. 250-253, 2008.

[23] J. M. Bioucas-Dias, "A variable splitting augmented Lagragian approach to linear spectral unmixing," IEEE GRSS Workshop Hyperspectral Image Signal Process.: Evolution in Remote Sens, pp. 1-4, 2009.

[24] M.-D. Iordache, J. Bioucas-Dias, and A. Plaza, "Sparse unmixing of hyperspectral data," IEEE Trans. Geosci. Remote Sens., vol. 49, no. 6, pp. 2014-2039, Jun. 2011.

[25] S. Zhang, J. Li, K. Liu, C. Deng, L. Liu, and A. Plaza, "Hyperspectral Unmixing Based on Local Collaborative Sparse Regression," IEEE Trans. Geosci. Remote Sens. Lett., vol. 13, no. 5, pp. 631-635, Mar. 2016.

[26] M.-D. Iordache, J. Bioucas-Dias, and A. Plaza, "Collaborative sparse regression for hyperspectral unmixing," IEEE Trans. Geosci. Remote Sens., vol. 52, no. 1, pp. 341-354, Jan. 2014.

[27] A. Marinoni, A. Plaza, and P. Gamba, "Harmonic Mixture Modeling for Efficient Nonlinear Hyperspectral Unmixing," IEEE Journal of Selected Topics in Applied Earth Observations and Remote Sensing, vol. 9, pp.4247-4256, 2016.

[28] J. Sevilla, L. I. Jiménez, and A. Plaza, "Sparse Unmixing-Based Content Retrieval of Hyperspectral Images on Graphics Processing Units," IEEE Trans. Geosci. Remote Sens. Lett., vol. 12, no. 12, pp. 2443-2447, Dec. 2015

[29] J. Delgado, G. Martín, J. Plaza, L. I. Jiménez, and A. Plaza, "Fast Spatial Preprocessing for Spectral Unmixing of Hyperspectral Data on Graphics Processing Units," IEEE Journal of Selected Topics in Applied Earth Observations and Remote Sensing, vol. 9, no. 2, pp.952-961, Feb. 2016.

[30] A. Halimi, Y. Altmann, N. Dobigeon, and J. Tourneret, "Unmixing hyperspectral images using the generalized bilinear model," in Proc. IEEE Int. Geosci. Remote Sens. Symp., pp. 1886-1889, Jul. 2011.

[31] N. Yokoya, J. Chanussot, and A. Iwasaki, "Nonlinear unmixing of hyperspectral data using semi-nonnegative matrix factorization," IEEE Trans. Geosci. Remote Sensing, vol. 52, no. 2, pp. 1430-1437, Feb. 2014

[32] X. Zhang, C. Cheng, J. An, Y. Zheng, E. Zhang, B. Hou, "Sparsity-constrained generalized bilinear model for hyperspectral unmixing," IEEE International Geoscience and Remote Sensing Symposium (IGARSS), pp. 5055-5058, 2015.

[33] W. f. Luo, L. r. Gao, A. Plaza, A. Marinoni, B. Y, L. Zhong, P. Gamba, and B. Z, "A New Algorithm for Bilinear Spectral Unmixing of Hyperspectral Images Using Particle Swarm Optimization," IEEE Journal of Selected Topics in Applied Earth Observations and Remote Sensing, pp.1-15, 2016.

[34] M. D. Iordache, A. Plaza, and J. Bioucas-Dias, "Recent developments in sparse hyperspectral unmixing," in Proc. IGARSS, pp. 1281-1284, 2010.

[35] R. Liu, B. Du, L. Zhang, "Hyperspectral Unmixing via Double Abundance Characteristics Constraints Based NMF," Remote Sens, vol. 8, no. 6, pp. 464, 2016

[36] W. He, H. Zhang, L. Zhang, "Sparsity-Regularized Robust Non-negative Matrix Factorization for Hyperspectral Unmixing," IEEE Journal of Selected Topics in Applied Earth Observations and Remote Sensing, vol. 13, no. 5, pp.686-690, 2016.

[37] J. Li, J. M. Bioucas-Dias, A. Plaza and L. Liu, "Robust Collaborative Nonnegative Matrix Factorization for Hyperspectral Unmixing," IEEE Transactions on Geoscience and Remote Sensing, accepted for publication, 2016

[38] L. Chen, J. Liu, et al., "Hyperspectral unmixing based on estimation of pixels mixing models," Infrared Technology, vol.38, no.2, pp.132-137, Feb. 2016.

[39] M. A. Veganzones, G. Tochon, M. Dalla-Mura, A. Plaza and J. Chanussot, "Hyperspectral Image Segmentation Using a New Spectral Unmixing-Based Binary Partition Tree Representation," IEEE Transactions on Image Processing, vol. 23, no. 8, pp: 3574 - 3589, 2014.

[40] J. M. P. Nascimento, and J. M. B. Dias, "Vertex component analysis: a fast algorithm to unmix hyperspectral data," IEEE Transactions on Geoscience and Remote Sensing, 43(4):898-910, 2005 
[41] X. Lu, H. Wu, Y. Yuan, et al., "Manifold Regularized Sparse NMF for Hyperspectral Unmixing," IEEE Transactions on Geoscience and Remote Sensing, 51(5):2815-2826, 2013.

[42] A. Halimi, Y. Altmann, N. Dobigeon, et al., "Unmixing hyperspectral images using the generalized bilinear model," IEEE International Geoscience and Remote Sensing Symposium, pp. 1886 - 1889, 2011.

[43] J. M. Bioucas-Dias and J. M. P. Nascimento, "Hyperspectral subspace identification," IEEE Trans. Geosci. Remote Sens., vol. 46, no. 8, pp. 2435-2445, Aug. 2008.

[44] Y. Qian, S. Jia, J. Zhou, and A. Robles-Kelly, "Hyperspectral unmixing via L1/2 sparsity-constrained nonnegative matrix factorization," IEEE Trans. Geosci.Remote Sensing, vol. 49, no. 11, pp. 4282-4297, 2011.

[45] N.Wang, B.Du, L. Zhang, “An Endmember Dissimilarity Constrained Non-Negative Matrix Factorization Method for Hyperspectral Unmixing," IEEE J. Sel. Top. Appl. Earth Obs. Remote Sens, pp. 554-569, Jun. 2013.

[46] D. C. Heinz and C.I. Chang, "Fully constrained least squares linear spectral mixture analysis method for material quantification in hyperspectral imagery," IEEE Trans. Geosci. Remote Sens., vol. 39, no. 3, pp. 529-545, Mar. 2001.
[47] N. Dobigeon, S. Moussaoui, M. Coulon, J. Y. Tourneret, and A. O. Hero, "Joint Bayesian endmember extraction and linear unmixing for hyperspectral imagery," IEEE Trans. Signal Process., vol. 57, no. 11, pp. 4355-4368, Nov. 2009.

[48] N. Dobigeon, J. Y. Tourneret, and C. I Chang, "Semi-supervised linear spectral unmixing using a hierarchical Bayesian model for hyperspectral imagery," IEEE Trans. Signal Process., vol. 56, no. 7, pp. 2684-2695, Jul. 2008.

[49] D. Iordache, J. Bioucas-Dias, and A. Plaza, "Collaborative sparse regression for hyperspectral unmixing," IEEE Trans. Geosci. Remote Sens., vol. 52, no. 1, pp. 341 - 354, Jan. 2014.

[50] X. Chen, and L. Vierling, Spectral mixture analyses of hyperspectral data acquired using a tethered ballon, Rem. Sens. of the Environ. 103, $338-350,2006$.

[51] J. M. P. Nascimentoa and J. M. Bioucas-Diasb, Nonlinear mixture model for hyperspectral unmixing, Proc. of SPIE, vol. 7477 74770I, 1-8, 2009.

[52] Q. Wang, J. Lin, Y. Yuan, Salient Band Selection for Hyperspectral Image Classification via Manifold Ranking, IEEE Trans Neural Netw Learn Syst. vol. 27, no. 6, pp. 1279-1289, Jun. 2016. 